\title{
Life-history evolution of class-structured populations in fluctuating environments
}

\author{
Sébastien Lion ${ }^{1}$ and Sylvain Gandon ${ }^{2}$
}

March 12, 2021

CEFE, CNRS, Univ Montpellier, EPHE, IRD, Univ Paul Valéry Montpellier 3. 1919, route de Mende, Montpellier, France

1. E-mail: sebastien.lion@cefe.cnrs.fr. ORCID: 0000-0002-4081-0038.

2. E-mail: sylvain.gandon@cefe.cnrs.fr. ORCID: 0000-0003-2624-7856.

Keywords: periodic fluctuations, reproductive value, selection gradient, adaptive dynamics, pathogen, virulence

\begin{abstract}
What is the influence of periodic environmental fluctuations on life-history evolution? We present a general theoretical framework to understand and predict the long-term evolution of lifehistory traits under a broad range of ecological scenarios. Indeed, this analysis yields time-varying selection gradients that help dissect the influence of the fluctuations of the environment on the competitive ability of a specific life-history mutation. We use this framework to analyse the evolution of key life-history traits of pathogens, such as transmission and virulence. These examples reveal how periodic fluctuations of the environment can affect the evolution of pathogens, and illustrate the usefulness and broad applicability of this new approach.
\end{abstract}

\section{Introduction}

Many organisms experience periodic fluctuations of their environment. These fluctuations may be driven by abiotic variations of the environment at different time scales (e.g. diurnal and seasonal variability), or by the dynamics of biotic interactions between organisms (e.g. predator-prey or hostparasite limit cycles). In such periodically changing environments, selection on life-history traits is likely to fluctuate over time, but we currently lack a good understanding of the feedback between periodic environmental dynamics and long-term phenotypic evolution (Barraquand et al., 2017).

A good measure of selection in periodic environments should tell us whether, on average over one period of the fluctuation, a mutation increases or decreases in frequency. But how should we compute this average fitness when selection may vary both in time but also among different classes of individuals? Floquet theory provides an answer to this question through the computation of the invasion fitness of a rare mutant in the periodic environment produced by the wild type (Metz et al., 1992; Meszéna et al., 2005; Klausmeier, 2008; Metz, 2008). However, the analysis based on Floquet theory is numerical and yields little biological insight. It only provides a good understanding of evolutionary dynamics when a single class of individuals is needed to describe the mutant dynamics. In this case the invasion fitness is simply the average, over one period, of the per-capita growth rate of a rare mutant (Metz, 2008; Donnelly et al., 2013; Kremer \& Klausmeier, 2013; Cornet et al., 2014; Gandon, 2016; Ferris \& Best, 2018; Pigeault et al., 2018). In class-structured populations, however, the lack of an analytical expression for the invasion fitness hampers the biological interpretation of 
the results obtained with Floquet analysis. In this paper, we fill this gap and provide a new method to analyse selection in class-structured populations subject to periodic environmental fluctuations.

Intuitively, one would expect that the direction of selection should depend on the relative abundance of each class as well as the productivity of the focal organism in each class. In constant environments, this classical distinction between the quantity and the quality of different classes has been shown to be biologically enlightening (Taylor, 1990; Taylor \& Frank, 1996; Gandon, 2004; Rousset, 2004; Lehmann \& Rousset, 2014; Lion, 2018a). Our new approach extends this distinction to periodic environments and allows us to derive, using only the standard weak-selection assumption, an analytical expression of the selection gradient in terms of the quantity and quality of classes, which are now time-dependent variables (see figure 1 for a graphical summary). With this approach, the selection gradients in periodic and constant environments are directly comparable and conceptually similar.

We first provide a general description of eco-evolutionary dynamics in class-structured, polymorphic populations, then turn to the dynamics of a mutant invading a resident population. We show that, under weak selection (that is, for mutations of small phenotypic effects), a separation of time scales argument can be used to derive the selection gradient in periodically varying environments. To illustrate the potential use of this approach, we focus on the evolution of pathogen life-history traits (such as transmission and virulence) when there is periodic variation in the availability of susceptible hosts. The focus on pathogens is not restrictive, and the method can be applied to a variety of life cycles. Evolutionary epidemiology, however, provides a very natural framework in which to think about potentially complex eco-evolutionary feedbacks.

\section{Eco-evolutionary dynamics}

We consider a focal population composed of $K$ different classes of individuals. For instance, these different classes may correspond to distinct developmental stages of the organism (e.g., young and old, male or female), different immune states, or different locations in a spatially structured environment. Because we are interested in evolution we also assume that the population is polymorphic and composed of $M$ different genotypes.

The life cycle is defined by a matrix of average transition rates, $\bar{r}^{k j}(\mathbf{E}, t)$, which refer to the net production of class- $k$ individuals by class $-j$ individuals, averaged over all genotypes (Lion, 2018a,b). These transitions can be due to reproduction, mortality, maturation, or dispersal depending on the biological context. Crucially, these rates can vary with a change in the environment which is referred to as $\mathbf{E}(t)$ (Metz et al., 1992; Metz, 2008; Lion, 2018b). These environmental variations may be driven by density-dependent effects caused by changes in population densities $\mathbf{n}(t)$, by frequencydependent effects caused by changes in the frequencies $\mathbf{p}(t)$ of the different types, but also by changes in extrinsic variables (such as the density of a resource) which we refer to as $\mathbf{e}(t)$, so that $\mathbf{E}(t)=$ $\left(\begin{array}{lll}\mathbf{n}(t) & \mathbf{p}(t) & \mathbf{e}(t)\end{array}\right)^{\top}$. Table 1 gives a summary of the main notations.

The average transition rates depend on the transition rates of the various genotypes, $r_{i}^{k j}(\mathbf{E}, t)$, and on the frequencies $f_{i}^{j}$ of genotype $i$ in class $j$. Thus, we have

$$
\bar{r}^{k j}(\mathbf{E}, t)=\sum_{i} r_{i}^{k j}(\mathbf{E}, t) f_{i}^{j}(t)
$$

Following Lion (2018a,b), this yields the following eco-evolutionary dynamics:

$$
\begin{aligned}
\frac{\mathrm{d} \mathbf{n}(t)}{\mathrm{d} t} & =\overline{\mathbf{R}}(\mathbf{E}, t) \mathbf{n}(t) \\
\frac{\mathrm{d} f_{i}^{k}(t)}{\mathrm{d} t} & =\sum_{j}\left(r_{i}^{k j}(\mathbf{E}, t) f_{i}^{j}(t)-\bar{r}^{k j}(\mathbf{E}, t) f_{i}^{k}(t)\right) \frac{f^{j}(t)}{f^{k}(t)}
\end{aligned}
$$

where $f^{j}(t)=n^{j}(t) / \sum_{k} n^{k}(t)$ is the fraction of individuals in class $j$ at time $t$, and $\overline{\mathbf{R}}(\mathbf{E}, t)$ is the matrix of average per-capita transition rates between different classes of individuals. Note that if the environment depends on extrinsic variables (e.g. the density of a resource or a predator), one needs to 
Table 1: Definition of the main mathematical symbols

\begin{tabular}{ll}
\hline Mathematical symbol & Description \\
\hline$n^{k}(t)$ & Density of individuals in class $k(1 \leq k \leq K)$ \\
$n(t)=\sum_{k} n^{k}(t)$ & Total density of individuals \\
$f^{k}(t)=n^{k}(t) / n(t)$ & Frequency of individuals in class $k$ (with respect to the total population) \\
$v^{k}(t)$ & Individual reproductive value for class $k$ \\
$\mathbf{n}(t)$ & Vector of class densities $n^{k}(\mathrm{t})$ \\
$\mathbf{f}(t)$ & Vector of class frequencies $f^{k}(\mathrm{t})$ \\
$\mathbf{v}(t)$ & Vector of individual reproductive values $v^{k}(t)$ \\
$\mathbf{e}(t)$ & Vector of external ecological variables \\
\hline$f_{i}^{k}(t)$ & Frequency of genotype $i$ within class $k(1 \leq i \leq M)$ \\
$\mathbf{p}(t)$ & Vector of genotype frequencies $f_{i}^{k}($ dimension $K \times M)$ \\
$f_{i}(t)=\sum_{k} f_{i}^{k}(t) f^{k}(t)$ & Global frequency of genotype $i$ \\
$\tilde{f}_{i}(t)=\sum_{k} v^{k}(t) f_{i}^{k}(t) f^{k}(t)$ & Reproductive-value-weighted frequency of genotype $i$ \\
\hline$r_{i}^{k j}$ & Per-capita transition rate of genotype- $i$ individuals from class $j$ to \\
$\bar{r}^{k j}=\sum_{i} r_{i}^{k j} f_{i}^{j}$ & class $k$ \\
$\overline{\mathbf{R}}$ & Average per-capita transition rate at from class $j$ to class $k$ \\
\hline
\end{tabular}

specify the dynamics of $\mathbf{e}(t)$ to complete the characterisation of the dynamical system (2). Thus, the eco-evolutionary dynamics are described by the $M(K+1)$ equations of system (2), plus the equations needed to describe the dynamics of extrinsic variables.

\subsection{Dynamics of mutant frequencies}

Now suppose that, for simplicity, we only have two types in the population: a resident wild type $(w)$ and a mutant $(m)$. The change in the global frequency of the mutant, $f_{m}=\sum_{k} f_{m}^{k} f^{k}$, can then be decomposed as follows (Day \& Gandon, 2006; Osnas et al., 2015; Lion \& Gandon, 2016; Lion, 2018a).

$$
\begin{aligned}
\frac{\mathrm{d} f_{m}}{\mathrm{~d} t}= & \underbrace{\sum_{j} f_{m}^{j}(t)\left(1-f_{m}^{j}(t)\right) \sum_{k}\left(r_{m}^{k j}(\mathbf{E}, t)-r_{w}^{k j}(\mathbf{E}, t)\right) f^{j}(t)}_{\text {within-class }} \\
& +\underbrace{\sum_{j}\left(f_{m}^{j}(t)-f_{m}(t)\right) \sum_{k} \bar{r}^{k j}(\mathbf{E}, t) f^{j}(t)}_{\text {between-class }} .
\end{aligned}
$$

The first line represents the average effect of within-class selection, given by the genetic variance $f_{m}^{j}\left(1-f_{m}^{j}\right)$ within class $j$ times the difference in transition rates from class $j$ to all other classes. The average is taken over the class distribution $f^{j}$. In contrast, the second line represents the effect of gene flow between classes, which depends on the relative contribution of the different classes when there is variation in genotype frequencies (differentiation) among classes. Importantly, this second term conflates both the effect of selection (which can shape the differentiation $f_{m}^{j}-f_{m}$ ) and of purely demographic processes (what Grafen (2015) termed "passive changes" in allele frequencies), so that any estimation of selection based on equation (3) may be biased by the existence of intrinsic differences in qualities between classes.

Thus, although there can be value in explicitly tracking the dynamics of the genetic differentiation between classes (see e.g. Berngruber et al. (2013), Berngruber et al. (2015), and Lion \& Gandon (2016)), it is for our purpose more convenient to use an alternative measure of the mutant frequency,

$$
\tilde{f}_{m}=\sum_{k} v^{k}(t) f_{m}^{k}(t) f^{k}(t)
$$

which weights the mutant frequency in class $k$ by the quantity $\left(f^{k}(t)\right)$ and quality $\left(v^{k}(t)\right)$ of individuals in class $k$ at time $t$. Specifically, we use the reproductive value of an individual in class $k$ at time $t$ 


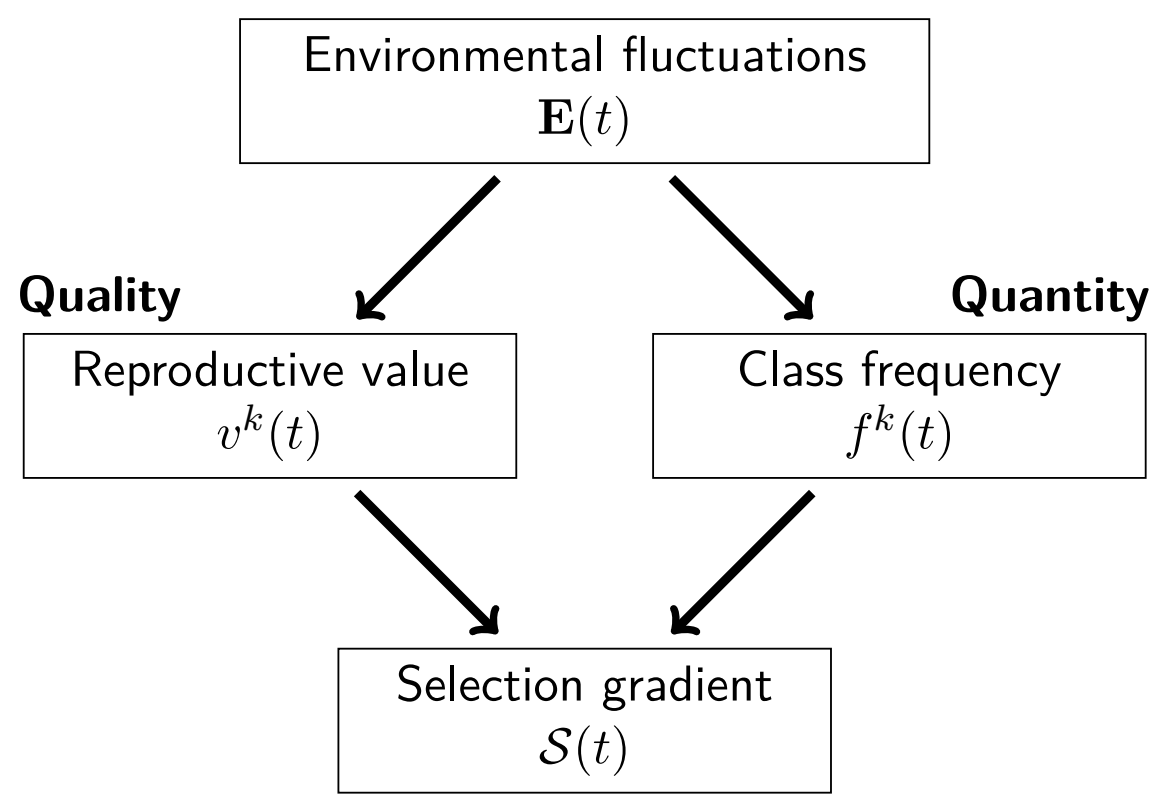

Figure 1: Graphical summary of our approach. Environmental fluctuations are used to evaluate the quality and quantity of individuals in the different classes at a given time. This information can then be used to calculate the selection gradient.

as the measure of quality $v^{k}(t)$. As previously shown (Lion, 2018a), the dynamics of this weighted average frequency can then be written as

$$
\frac{\mathrm{d} \tilde{f}_{m}}{\mathrm{~d} t}=\sum_{j} \underbrace{f_{m}^{j}(t)\left(1-f_{m}^{j}(t)\right)}_{\text {genetic variance }} \sum_{k} v^{k}(t)\left(r_{m}^{k j}(\mathbf{E}, t)-r_{w}^{k j}(\mathbf{E}, t)\right) f^{j}(t) .
$$

In contrast to equation (3), equation (5) describes the net effect of selection on the change in mutant frequency, without the confounding variations due to "passive changes". It thus provides a more convenient measure of selection where the overall change in mutant frequency is driven by the sum of the effects of the mutation on the rates $r^{k j}$ weighted by the frequency of class $j$ and the individual reproductive value of class $k$. Crucially, both the quantity and the quality of the different classes can change in a fluctuating environment, so, unlike classical equilibrium theory we need to characterise these fluctuations to understand and predict life-history evolution.

\subsection{Dynamics of class frequencies}

Following Lion (2018a), the class frequencies follow the dynamics:

$$
\frac{\mathrm{d} \mathbf{f}}{\mathrm{d} t}=\overline{\mathbf{R}}(\mathbf{E}, t) \mathbf{f}(t)-\bar{r}(\mathbf{E}, t) \mathbf{f}(t),
$$

where $\bar{r}(\mathbf{E}, t)=\sum_{k} \sum_{j} \bar{r}^{k j}(\mathbf{E}, t) f^{j}(t)$ is the average per-capita growth rate of the population. If we focus on the frequency of class $j$ we have:

$$
\frac{\mathrm{d} f^{j}}{\mathrm{~d} t}=\sum_{k} \bar{r}^{j k}(\mathbf{E}, t) f^{k}(t)-\bar{r}(\mathbf{E}, t) f^{j}(t) .
$$

Hence, the change in frequency of class $j$ depends on the current class frequencies and on the relative contributions of all the classes to class $j$. Note that we need to account for the overall growth rate $\bar{r}$ of the whole population. This is because we are monitoring the change in class frequencies, not class densities. The sum of class densities can increase (i.e. when $\bar{r}>0$ ) but the sum of class frequencies must remain equal to 1 at all times (i.e. $\sum_{i} f^{i}(t)=1$ ).

Mathematically, we can calculate the class frequencies at time $t$ by integrating equation (6) forward in time from an appropriate initial condition (i.e. an initial condition that leads to a biologically relevant periodic attractor after some time). In other words, the quantity of class $j$ at time $t$ depends on the past trajectory of the population. 


\subsection{Dynamics of individual reproductive values}

Similarly, the individual reproductive values, collected in the row vector $\mathbf{v}^{\top}(t)$, follow the dynamics (Lion, 2018a):

$$
\frac{\mathrm{d} \mathbf{v}^{\top}}{\mathrm{d} t}=-\mathbf{v}^{\top}(t) \overline{\mathbf{R}}(\mathbf{E}, t)+\bar{r}(\mathbf{E}, t) \mathbf{v}^{\top}(t)
$$

If we focus on the individual reproductive value in class $k$, we have:

$$
\frac{\mathrm{d} v^{k}}{\mathrm{~d} t}=-\sum_{j} v^{j}(t) \bar{r}^{j k}(\mathbf{E}, t)+\bar{r} v^{k}(t) .
$$

Mathematically, equation (8) is the adjoint of equation (6), which generalises the fact that, in constant environments, class frequencies and individual reproductive values are respectively right and left eigenvectors of the transition matrix $\overline{\mathbf{R}}$. Biologically, $v^{k}(t)$ gives the quality of class $k$ at time $t$, as measured by the fraction of future descendants of an individual in class $k$ from time $t$ onwards, relative to an average individual in the population.

As in equation (6), we take into account the change in total population size (through $\bar{r}$ ) so that $\mathbf{f}$ and $\mathbf{v}^{\top}$ are co-normalised at all times (i.e. $\mathbf{v}^{\top}(t) \mathbf{f}(t)=1$ ). In other words, the reproductive value of an average individual, $\sum_{k} v^{k}(t) f^{k}(t)$ is equal to 1 at all times, and $v^{k}(t)$ measures the contribution to the future of the population of an individual sampled in class $k$ at time $t$, relative to a randomly sampled individual.

Hence, measuring the quality of a class at time $t$ depends on the descendants and thus on the future of the population. In contrast with class frequencies, we compute the individual reproductive values by integrating equation (8) backward in time, from a terminal condition at a time $t_{f}$ in the distant future. Typically, we set $v^{k}\left(t_{f}\right)=1$ for all classes at time $t_{f}$ (Barton \& Etheridge, 2011; Lion, 2018a). This terminal condition indicates that the relative qualities of the different classes of individuals are unknown at that time and we use between-class transitions to acquire information on these relative qualities on the periodic attractor, by integrating the system (8) backwards.

\section{Weak selection and the selection gradient}

In this section, we use a weak-selection approximation of equation (5) to derive a novel analytical expression of the selection gradient using dynamical reproductive values. Our approach is based on a separation of time scales, which readily occurs when the mutant phenotype, $z_{m}$, is close to the resident phenotype $z_{w}$, so that $z_{m}=z_{w}+\varepsilon$, where $\varepsilon$ is small. Under this separation of time scales, the population will quickly settle on a population dynamical attractor, such as a fixed point or a limit cycle, and we can use equations (6) and (8) to compute the quality and quantity of each class at any given time on the attractor. The fixed point case corresponds to the classical theory developed for constant environments (Taylor, 1990; Rousset, 2004; Otto \& Day, 2007), and the limit cycle case corresponds to an extension of this theory to periodic population dynamics, which we now present.

In appendix $\mathrm{A}$, we show that the class densities $\mathbf{n}(t)$, class frequencies $\mathbf{f}(t)$ and individual reproductive values $\mathbf{v}^{\top}(t)$ are fast variables. This means that, under weak selection, the environment $\mathbf{E}(t)$ quickly settles on a periodic attractor which is well approximated by the attractor of a monomorphic resident population, $\hat{\mathbf{E}}(t)$. On this monomorphic attractor, we only need the densities $\hat{\mathbf{n}}(t)$ and the external variables $\hat{\mathbf{e}}(t)$ to fully characterise the environment experienced by the mutant type.

In contrast, the weighted mutant frequency $\tilde{f}_{m}$ is a slow variable. To first-order in $\varepsilon$, we can write the dynamics of $\tilde{f}_{m}$ as

$$
\frac{\mathrm{d} \tilde{f}_{m}}{\mathrm{~d} t}=\varepsilon \tilde{f}_{m}\left(1-\tilde{f}_{m}\right) \mathcal{S}(t)+O\left(\varepsilon^{2}\right)
$$

where the instantaneous selection gradient is given by

$$
\mathcal{S}(t)=\left.\sum_{j} \sum_{k} \hat{v}^{k}(t) \frac{\partial r_{m}^{k j}(\hat{\mathbf{E}}, t)}{\partial z_{m}}\right|_{z_{m}=z_{w}} \hat{f}^{j}(t) .
$$


As a result of periodic fluctuations on the fast time scale, the value and sign of the selection gradient may fluctuate. However, under weak selection, these fast fluctuations can be ironed out. This is known as the averaging principle (Cai \& Geritz, 2020), which allows us to approximate the dynamics of the mutant frequency on the slow time scales by the solution of the so-called averaged system

$$
\frac{\mathrm{d} \tilde{f}_{m}}{\mathrm{~d} t}=\varepsilon \tilde{f}_{m}\left(1-\tilde{f}_{m}\right) \mathcal{S}
$$

where

$$
\mathcal{S}=\left\langle\left.\sum_{j} \sum_{k} \hat{v}^{k}(t) \frac{\partial r_{m}^{k j}(\hat{\mathbf{E}}, t)}{\partial z_{m}}\right|_{z_{m}=z_{w}} \hat{f}^{j}(t)\right\rangle
$$

is the selection gradient averaged over one period of the resident attractor (i.e. $\langle X\rangle=\frac{1}{T} \int_{\tau}^{\tau+T} X(t) \mathrm{d} t$ for any given $\tau$ on the periodic attractor).

The selection gradient (13) takes the form of a sum of marginal selective effects (giving the influence of the evolving trait on between-class transitions) weighted by the time-varying quantity and quality of different classes and is therefore reminiscent of the expressions obtained in equilibrium classstructured populations. In fact, for constant environments, the class frequencies, reproductive values and environment are constant, and therefore equation (13) exactly reduces to the classical expression of the selection gradient for class-structured equilibrium populations (Taylor, 1990; Rousset, 2004; Otto \& Day, 2007). For periodic environments, equation (13) provides an analytical approximation, for weak selection, of the invasion fitness of a mutant typically calculated as a Floquet exponent (Appendix B). Note that this first-order approximation gives information on the direction of selection and its potential evolutionary endpoints, but not on the evolutionarily stability of these singularities, for which a numerical computation of the Floquet exponent is still needed (Appendix B).

Importantly, all the fast variables in equation (13) are evaluated on the attractor of the monomorphic resident population. Although in general these solutions can only be computed numerically, we show in section 4 that algebraic manipulations of equation (13) can be used to shed light on the interplay between fluctuating environmental dynamics and selection. Hence, equation (13) is the central result of our article and leads to a general recipe to study the evolution of life-history traits in class-structured populations experiencing periodic environmental fluctuations. The main steps of this method are summarised in Box 1. In the next section, we apply our general method to the evolution of pathogen life-history traits when the density of hosts fluctuates.

\section{Pathogen evolution in fluctuating environments}

Many pathogens have to cope with environmental fluctuations (Altizer et al., 2006; Martinez, 2018). In this section, we use the approach in Box 1 to study pathogen evolution under three distinct epidemiological scenarios corresponding to three different pathogen life cycles (we will follow the steps described in Box 1). In all scenarios the pathogen can be present in two distinct classes of hosts $(A$ and $B$ ) and the environmental fluctuations are captured by a periodic function $\nu(t)$ that gives the probability of production of susceptible hosts at time $t$. Hence, using our general terminology, the forcing function $\nu(t)$ causes periodic fluctuations in the vector $\mathbf{e}(t)$ (collecting the densities of susceptible hosts), which in turn drives fluctuations in the vector $\mathbf{n}(t)$ (collecting the densities of infected hosts in classes $A$ and $B$ ). The general approach outlined in Box $\mathbf{1}$ can be applied to any periodic function, but for simplicity we consider a smooth version of a step function with minimum 0 , maximum $\nu_{\max }$, and mean $\nu_{\max } / 2$ (figure 2).

\subsection{Scenario 1: the Curse of the Pharaoh hypothesis}

The claim that long-lived pathogen propagules could select for higher pathogen virulence has often been presented as the "curse of the pharaoh hypothesis" (Bonhoeffer et al., 1996; Gandon, 1998). Previous theoretical analyses focused mainly on temporally constant environments. Here we want to analyse the influence of fluctuations on the availability of susceptible hosts on the evolution of virulence for pathogens with free-living stages. 
bioRxiv preprint doi: https://doi.org/10.1101/2021.03.12.435065; this version posted March 12,2021 . The copyright holder for this preprint (which was not certified by peer review) is the author/funder, who has granted bioRxiv a license to display the preprint in perpetuity. It is made available under aCC-BY-NC-ND 4.0 International license.

\section{Box 1: How to study life-history evolution in periodically fluctuating environments?}

We detail below the different steps allowing us to derive the selection gradient (near the resident strategy) driving life-history evolution in a class-structured population in a fluctuating environment.

Step 1 - Formalise the description of the life cycle of the focal organism in a monomorphic resident population. This is captured in the dynamical system:

$$
\frac{\mathrm{d} \mathbf{n}}{\mathrm{d} t}=\mathbf{R n},
$$

where $\mathbf{R}$ is the matrix of between-class transition rates in the resident population, together with the dynamical equations for the extrinsic variables in $\mathbf{e}(t)$.

Step 2 - Determine which life-history traits are under selection (and the trade-off between these traits). Indeed, multiple life-history traits may be involved in the life-cycle and it is key to be very explicit about the constraints acting on the evolving traits.

Step 3 - Derive the selection gradient using equation (13). This step may often yield insight through the decomposition of selection into biologically meaningful quantities (see section 4 for examples). But in general, moving to steps 4 and 5 is needed to predict the influence of periodic fluctuations on life-history evolution.

Step 4 - Use equation (A) to determine the periodic attractor of the resident population. This will yield the forward dynamics of class frequencies.

Step 5 - Use equation (8) and the numerical solution derived in step 4 to solve the backward dynamics of individual reproductive values (using a final condition where individual reproductive values are all equal to 1 ).

Step 6 - The results of steps 4 and 5 can then be plugged into the selection gradient (step 3) to obtain an analytical expression or numerical approximation of the selective forces acting on the trait. This can be used to identify evolutionary singularities, the evolutionarily stability of which can be checked using Floquet analysis (Appendix B).

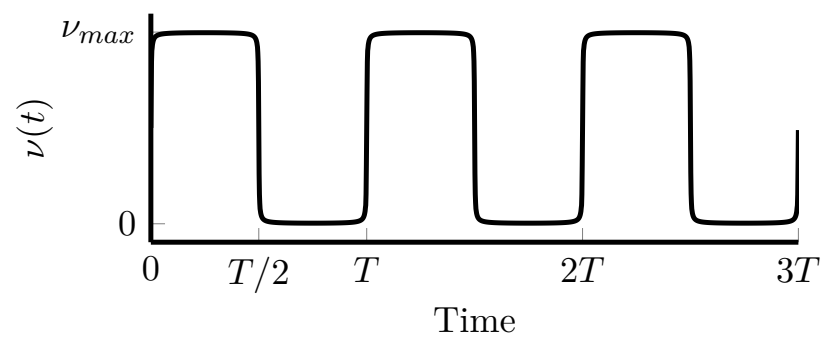

Figure 2: Environmental fluctuation. We model fluctuations in the environment with a smoothed step function with minimum 0 , maximum $\nu_{\max }$ and mean $\nu_{\max } / 2$. This is captured by the following function: $\nu(t)=\left(\nu_{\max } / 2\right)(1+(2 \zeta / \pi) \arctan (\sin (2 \pi t / T) / \delta)$. We typically use $\zeta=1$ and $\delta=0.01$. Note that $\zeta=0$ corresponds to a constant environment with value $\nu=\nu_{\max } / 2$. 
Let us assume that there is a single class of susceptible hosts $S$ but two different classes of the pathogens, which are (i) the infected host $\left(I^{A}\right)$ and (ii) the propagule stage (which lives outside the infected host but that we still note $I^{B}$ for consistency with the general framework): Thus, the vector of population densities is $\mathbf{n}(t)=\left(I^{A}(t) \quad I^{B}(t)\right)$ and $S(t)$ is the extrinsic environmental variable $\mathbf{e}(t)$. The epidemiological dynamics are given by

$$
\begin{aligned}
\frac{\mathrm{d} S}{\mathrm{~d} t} & =\theta(t)-d S(t)-\beta^{B} S(t) I^{B}(t) \\
\frac{\mathrm{d} I^{A}}{\mathrm{~d} t} & =\beta^{B} S(t) I^{B}(t)-\left(d^{A}+\alpha^{A}\right) I^{A}(t) \\
\frac{\mathrm{d} I^{B}}{\mathrm{~d} t} & =\beta^{A} I^{A}(t)-d^{B} I^{B}(t)
\end{aligned}
$$

where $\theta(t)=b \nu(t)$ refers to the periodic influx of susceptible hosts in the population. A susceptible host becomes infected upon contact with the propagules, which occurs at rate $\beta^{B} I^{B}(t)$. Infected hosts die at rate $d^{A}+\alpha^{A}$, where $\alpha^{A}$ represents virulence, and produce propagules at rate $\beta^{A}$. These propagules die at a rate $d_{B}$. Next, we follow the steps presented in Box 1 to explore how higher propagule survival (e.g. lower values of $d_{B}$ ) can affect the evolution of pathogen virulence $\alpha^{A}$ in the infected host.

Step 1: We can use equations (14) to derive the matrix $\mathbf{R}$ which captures the transition rates between classes $A$ and $B$ in a monomorphic resident population:

$$
\mathbf{R}=\left(\begin{array}{cc}
-d^{A}-\alpha^{A} & \beta^{B} S(t) \\
\beta^{A} & -d^{B}
\end{array}\right)
$$

Step 2: At this stage it is important to specify the constraints acting on the underlying trait $z$ that controls the evolution of pathogen virulence $\alpha^{A}$. As in previous studies, we assume that an increase in virulence is associated with an increase in pathogen transmission rate and in particular on the production of propagules $\beta^{A}$ (Bonhoeffer et al., 1996; Day \& Gandon, 2006).

Step 3: We use equation (11) to obtain the instantaneous selection gradient on the trait $z$ :

$$
\mathcal{S}(t)=f^{A}(t)\left(v^{B}(t) \frac{\mathrm{d} \beta^{A}}{\mathrm{~d} z}-v^{A}(t) \frac{\mathrm{d} \alpha^{A}}{\mathrm{~d} z}\right) .
$$

The first term between brackets represents the gain in fitness if a pathogen in class $A$ invests in the production of propagules (weighted by the individual reproductive value $v^{B}(t)$ of propagules). The second term between brackets accounts for the loss in fitness if a pathogen in class $A$ dies (weighted by the individual reproductive value $v^{A}(t)$ ). The selection gradient on $z$ is obtained by integrating (16) over one period of the fluctuation which yields:

$$
\mathcal{S}=\left\langle v^{B} f^{A}\right\rangle \frac{\mathrm{d} \beta^{A}}{\mathrm{~d} z}-\left\langle v^{A} f^{A}\right\rangle \frac{\mathrm{d} \alpha^{A}}{\mathrm{~d} z}
$$

An analysis of the dynamics of class frequencies $\mathbf{f}(t)$ and individual reproductive values $\mathbf{v}(t)$ is required to better understand selection on virulence and transmission.

Steps 4 and 5: For our life cycle, equation (8), which gives the dynamics of reproductive values, can be written as:

$$
\begin{aligned}
& \frac{\mathrm{d} v^{A}}{\mathrm{~d} t}=-\beta^{A} v^{B}(t)+\left(d^{A}+\alpha^{A}\right) v^{A}(t)+\bar{r} v^{A}(t), \\
& \frac{\mathrm{d} v^{B}}{\mathrm{~d} t}=-\beta^{B} S(t) v^{A}(t)+d^{B} v^{B}(t)+\bar{r} v^{B}(t) .
\end{aligned}
$$


Fluctuations in the availability of susceptible hosts cause fluctuations of the reproductive values which can be obtained numerically from (18), using the solution of system (14) to evaluate $S(t)$ and $\bar{r}(t)$. But equation (18) also yields a useful expression for the average of the ratio of individual reproductive values (Appendix S.1):

$$
\left\langle\frac{v^{B}}{v^{A}}\right\rangle=\frac{d^{A}+\alpha^{A}}{\beta^{A}}
$$

In other words, the mean value of this ratio is equal to the ratio of individual reproductive values in the absence of fluctuations.

Step 6: Equation (19) can be used to rewrite the selection gradient (17) which yields:

$$
\mathcal{S}=\left\langle c^{A}\right\rangle \mathcal{S}_{c}+\frac{\mathrm{d} \beta^{A}}{\mathrm{~d} z} \operatorname{Cov}\left(c^{A}(t), \frac{v^{B}(t)}{v^{A}(t)}\right)
$$

where $c^{A}(t)=v^{A}(t) f^{A}(t)$ is the class reproductive value at time $t$ and

$$
\mathcal{S}_{c}=\frac{d+\alpha^{A}}{\beta^{A}} \frac{\mathrm{d} \beta^{A}}{\mathrm{~d} z}-\frac{\mathrm{d} \alpha^{A}}{\mathrm{~d} z}
$$

refers to the gradient of selection in the absence of fluctuations. Equation (20) is particularly insightful because it shows how fluctuations of the availability of susceptible hosts can affect the evolution of the pathogen. Fluctuations only act on the evolution of the pathogen via the temporal covariance between $c^{A}(t)$ and $\frac{v^{B}(t)}{v^{A}(t)}$. In words, this means that, if infected hosts tend to be more abundant at times where propagules are relatively more valuable (e.g. the covariance is positive), the ES virulence will be higher than in a constant environment because the pathogen then reaps fitness benefits from increased propagule production.

Numerical simulations show that this covariance is expected to be positive when $d^{B}$ is low and negative when $d^{B}$ is large (figures $3 \mathrm{~b}-3 \mathrm{~d}$; see the SOM for an attempt to understand the sign of this covariance). In contrast with the analysis of Bonhoeffer et al. (1996) we thus expect fluctuations to alter the predictions regarding the influence of $d^{B}$ on the evolution of virulence and transmission rates. As shown in figure 3c, higher rates of survival (i.e. lower values of $d^{B}$ ) tend to select for higher virulence and transmission (as in the curse of the pharaoh hypothesis). However, this effect is non-monotonic: when $d^{B}$ gets very low (i.e. when propagule live very long), the covariance vanishes because $f^{A} \rightarrow 0$ and thus $c^{A} \rightarrow 0$, and therefore the ES virulence is the same as predicted in a constant environment. Note that Figure 3c also shows that, as expected, the ESS predicted from the selection gradient using time-dependent reproductive values is consistent with the value predicted from a more standard Floquet analysis (dashed line).

\subsection{Scenario 2: host preference}

Many pathogens can infect several host species. Intuitively, which host the pathogen should prefer will depend on the relative qualities of the hosts. But what would be the best strategy when the qualities or abundances of the different host species fluctuate? In our second scenario, we investigate the effect of periodic fluctuations in the abundance of different host species on the evolution of the pathogen's preference strategy.

We consider an epidemiological model with contact transmission (no free-living propagules) and assume that the pathogen can choose between two different hosts $(A$ or $B)$. When a pathogen enters a susceptible host $S^{A}$ (resp. $S^{B}$ ), we assume that the infection is successful with probability $p^{A}$ (resp. 
(a) $d^{B}=1$

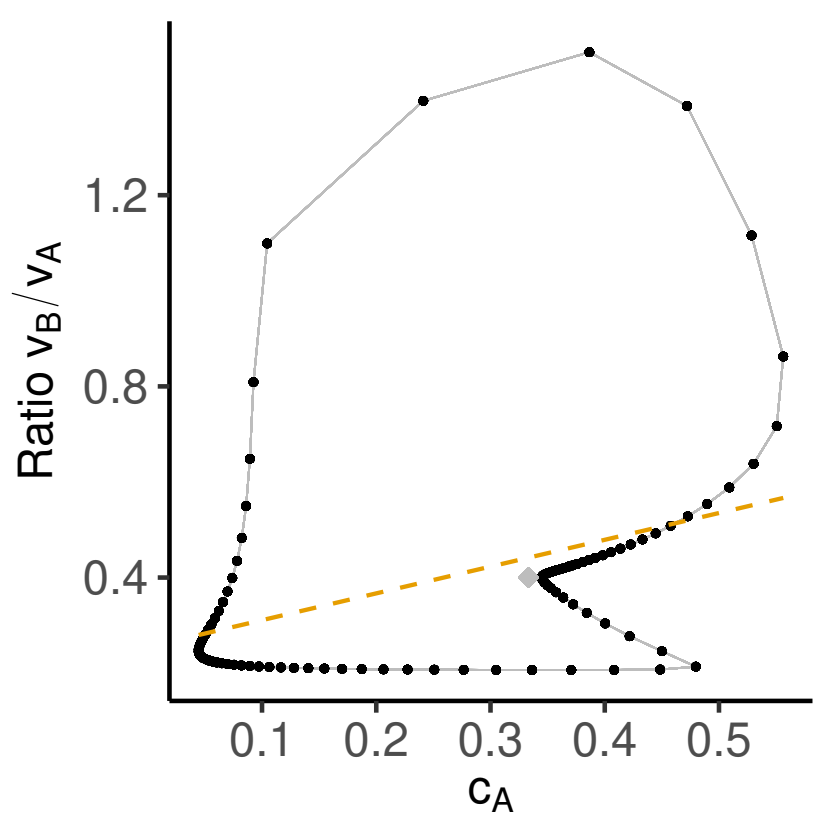

(b) $d^{B}=3$

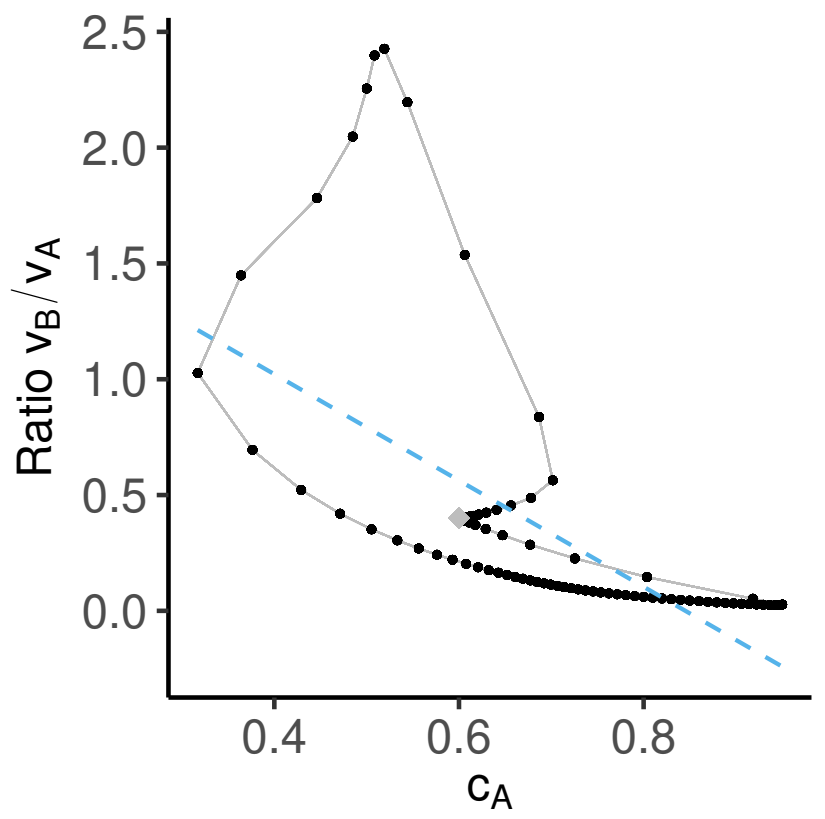

(c)

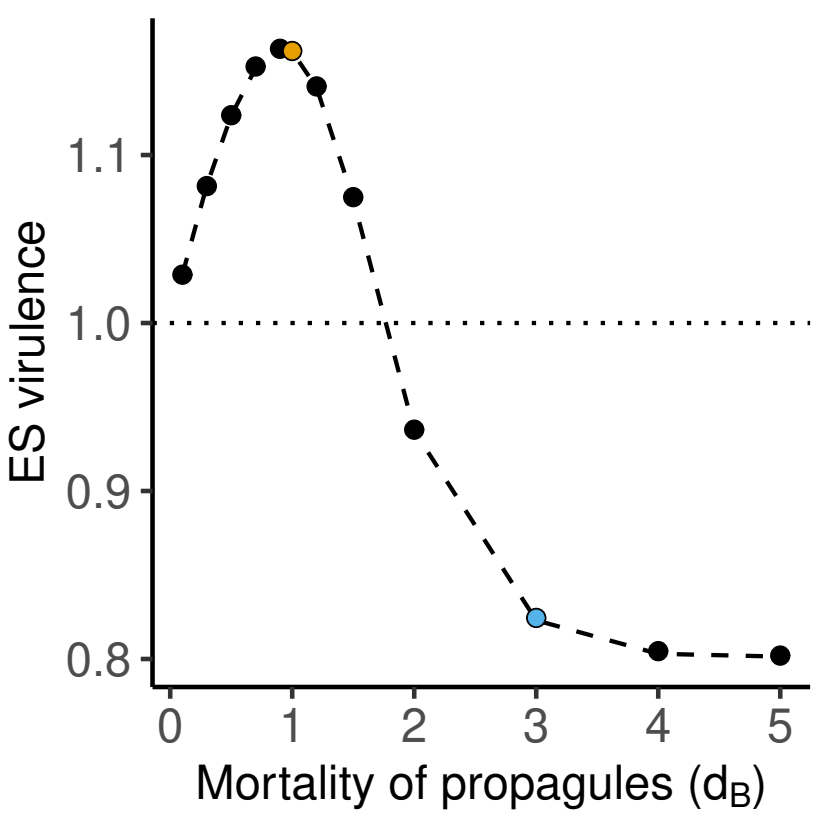

(d)

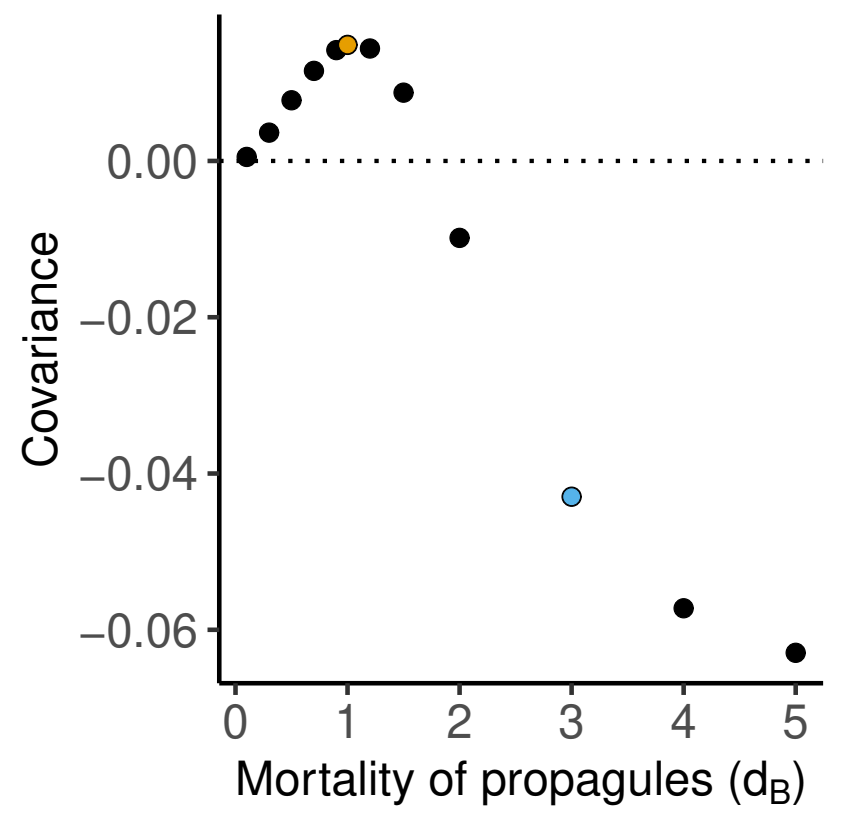

Figure 3: Scenario 1: The Curse of Pharaoh. (a) Parametric plot of $v^{B}(t) / v^{A}(t)$ versus $c^{A}(t)$ for $d^{B}=1$ and $z_{w}=z_{e q}$. The grey diamond gives the equilibrium for a constant environment. The slope of the regression line (dashed) is proportional to the temporal covariance between $c^{A}(t)$ and $v^{B}(t) / v^{A}(t)$. (b) Same as (a) but with $d^{B}=3$. (c) Predicted ESS as a function of the mortality rate of propagules, $d^{B}$ using the reproductive-value-based approach (dots) and the Floquet exponent (dashed line). The dotted line gives the prediction of the corresponding equilibrium model, $z_{e q}=1$. (d) Mean value of the temporal covariance between the class reproductive value $c^{A}(t)$ and the ratio of individual reproductive values $v^{B}(t) / v^{A}(t)$ as a function of $d^{B}$ for $z_{w}=z_{e q}$. Parameters: $\nu(t)=$ $0.5(1+(2 / \pi) \arctan (\sin (2 \pi t / T) / 0.01)), b=8, d=d^{A}=1, \beta^{A}(z)=\beta_{0} z /(1+z), \beta^{B}=\beta_{0}=10$, $\alpha^{A}(z)=z, T=10$. 
$\left.p^{B}\right)$. With these assumptions, the dynamical system becomes:

$$
\begin{aligned}
\frac{\mathrm{d} S^{A}}{\mathrm{~d} t} & =\theta^{A}(t)-d^{A} S(t)-h(t) p^{A} S(t) \\
\frac{\mathrm{d} S^{B}}{\mathrm{~d} t} & =\theta^{B}(t)-d^{B} S(t)-h(t) p^{B} S(t) \\
\frac{\mathrm{d} I^{A}}{\mathrm{~d} t} & =h(t) p^{A} S^{A}(t)-\left(d^{A}+\alpha^{A}\right) I^{A}(t) \\
\frac{\mathrm{d} I^{B}}{\mathrm{~d} t} & =h(t) p^{B} S^{B}(t)-\left(d^{B}+\alpha^{B}\right) I^{B}(t)
\end{aligned}
$$

where $h(t)=\beta^{A} I^{A}(t)+\beta^{B} I^{B}(t)$ is the force of infection. The two infection routes differ by their epidemiological parameters, so that one infection route may be more contagious or virulent than the other. Furthermore, we assume that the production of susceptible hosts is periodic, and such that $\theta^{A}(t)=b \nu(t)$ and $\theta^{B}=b(1-\nu(t))$, where $\nu(t)$ is the probability of production of $A$ hosts (figure 2).

Step 1: From equations (21a), we derive the transition matrix

$$
\mathbf{R}=\left(\begin{array}{cc}
\beta^{A} p^{A} S^{A}(t)-\left(d^{A}+\alpha^{A}\right) & \beta^{B} p^{A} S^{A}(t) \\
\beta^{A} p^{B} S^{B}(t) & \beta^{B} p^{B} S^{B}(t)-\left(d^{B}+\alpha^{B}\right)
\end{array}\right)
$$

Step 2: We assume that there is a trade-off between $p^{A}$ and $p^{B}$ such that $p^{A}=z=1-p^{B}$. Hence, if $z=1$, infection is only possible on $A$ hosts, while if $z=1 / 2$, both host classes are equally susceptible to infection. The trait $z$ can thus be interpreted as measuring preference towards $A$ hosts. For simplicity, we assume that the pathogen's survival is lower in host $A$, but its transmissibility is independent of the host (i.e. $\beta^{A}=\beta^{B}=\beta$, but $\alpha^{A}>\alpha^{B}$ ).

Step 3: Based on these assumptions on the life cycle, a naive prediction could be that the pathogen should always prefer the "good" host $B$, in which it enjoys a longer lifespan. However, the optimal strategy depends on the relative availability of the two classes of hosts, which can fluctuate over time. To better understand the selective pressures on the preference trait, we use our approach to derive the selection gradient and obtain:

$$
\mathcal{S}(t)=\beta\left[v^{A}(t) S^{A}(t)-v^{B}(t) S^{B}(t)\right]
$$

The terms $v^{k} S^{k}(t)$ have a simple interpretation as the expected reproductive output of a pathogen propagule at time $t$ through class $k$. Thus, the direction of selection is determined by whether this reproductive output is larger through class $A$ than through class $B$. Furthermore, potential evolutionary endpoints satisfy the balance condition

$$
\left\langle v^{A} S^{A}\right\rangle=\left\langle v^{B} S^{B}\right\rangle
$$

which simply states that selection halts when average reproductive outputs are the same in both classes.

Steps 4 and 5: To better understand the impact of periodic fluctuations in host availability on the evolution of the preference trait, we now need to numerically calculate the dynamics of the reproductive values and densities of susceptible hosts. A detailed discussion of these dynamics is given in the SOM, but we show in figures $4 \mathrm{a}-4 \mathrm{~b}$ that the period of $\nu(t)$ has a strong impact on the dynamics of the difference in reproductive output $D(t)=v^{A}(t) S^{A}(t)-v^{B}(t) S^{B}(t)$. When the period is small (figure $4 a), D$ fluctuates rapidly around a mean that is close to its value in a constant environment. In contrast, for large periods (figure $4 \mathrm{~b}$ ), $D(t)$ better tracks the environmental fluctuations $\nu(t)$, and its mean $\langle D\rangle$ is higher. 
Step 6: Using the dynamics of the ecological variables, it is possible to calculate the change in frequency (Appendix S.2) and the ES strategy for different values of T. Figure 4c shows that the ESS increases with the period of fluctuations: for small periods, the ecological variables fluctuate rapidly around their equilibrium values and as a result the ESS is close to the prediction of the equilibrium model. As the period of fluctuations increases, the average environment perceived by the pathogen becomes more different from the equilibrium environment, and the pathogen spends more time around high densities of $S^{A}$ hosts, which makes the $A$ hosts on average more valuable. In the limit of large periods, the ESS only depends on the basic reproductive ratios in each class, $R^{k}=\beta^{k} /\left(d+\alpha^{k}\right)$. Indeed, $D(t)$ tends towards $\left(1 /\left(p^{A} R^{A}\right)-\left(1 /\left(p^{B} R^{B}\right)\right)\right) / 2$ (Appendix $\mathrm{S} .2$, figure $4 \mathrm{~b}$ ). This yields the upper limit $z_{\infty}^{*}=R^{B} /\left(R^{A}+R^{B}\right)$ for large periods.

\subsection{Scenario 3: imperfect vaccines}

The use of imperfect vaccines may affect the evolution of pathogen virulence and transmission. These evolutionary consequences have been studied by Gandon et al. (2001, 2003) when the coverage of vaccination does not fluctuate in time. In our third epidemiological scenario, we ask how fluctuations in vaccination coverage may affect the evolution of virulence, building on a recent study by Walter \& Lion (2021).

We consider the same epidemiological dynamics as in Scenario 2, but assume that hosts are inoculated at birth with an imperfect vaccine at a rate $\nu(t)$ that fluctuates periodically (figure 2 ). This yields a fluctuating influx of $A$ hosts that are unvaccinated $\left(\theta^{A}(t)=b(1-\nu(t))\right)$ and $B$ hosts that are vaccinated $\left(\theta^{B}(t)=b \nu(t)\right)$.

Steps 1 and 2: As in scenario 2, the transition matrix $\mathbf{R}$ is given by equation (22). We now assume that $p_{A}=p_{B}=1$, and consider that the vaccine can either act by reducing the transmissibility of hosts $B\left(\beta^{B}=\left(1-r_{b}\right) \beta^{A}\right)$ or by decreasing virulence $\left(\alpha^{B}=\left(1-r_{a}\right) \alpha^{A}\right)$. This corresponds to the antitransmission and anti-toxin vaccines introduced by Gandon et al. (2001) (noted $r_{3}$ and $r_{4}$ respectively in that paper). Finally, we assume that, as in Scenario 2, the trait under selection is the pathogen strategy of host exploitation, $z$, and that transmission and virulence both depend on $z$.

Step 3: With these assumptions, the selection gradient at time $t$ takes the form

$$
\mathcal{S}(t)=c^{A}(t)\left[\frac{\mathrm{d} \beta^{A}}{\mathrm{~d} z} \omega^{A}(t)-\frac{\mathrm{d} \alpha^{A}}{\mathrm{~d} z}\right]+c^{B}(t)\left[\frac{\mathrm{d} \beta^{B}}{\mathrm{~d} z} \omega^{B}(t)-\frac{\mathrm{d} \alpha^{B}}{\mathrm{~d} z}\right]
$$

where $c^{A}(t)$ and $c^{B}(t)=1-c^{A}(t)$ are the class reproductive values in class $A$ and $B$ respectively, and

$$
\omega^{k}(t)=\frac{v^{A}(t) S^{A}(t)+v^{B}(t) S^{B}(t)}{v^{k}(t)} .
$$

Note that $\omega^{k}(t)$ has a useful intepretation: the denominator gives the quality of an "adult" pathogen in class $k$, while the numerator is the expected quality of a pathogen propagule and therefore quantifies the reproductive value of an "offspring" pathogen. So $\omega^{k}(t)$ gives a measure of how valuable reproduction is compared to survival in class $k$ at any given time.

Steps 4, 5 and 6: In general, the densities and reproductive values have complex periodic dynamics (figures S.3 and S.4). However, using the dynamics of reproductive values, it is possible to analytically show (Appendix S.3) that, in the resident population on its periodic attractor:

$$
\left\langle\omega^{k}\right\rangle=\frac{d+\alpha^{k}}{\beta^{k}}
$$

The term on the right-hand-side is one over the basic reproduction ratio $R^{k}$ of a pathogen in class $k$, and corresponds to the equilibrium value in a model with constant vaccination coverage. 
(a) $T=1$

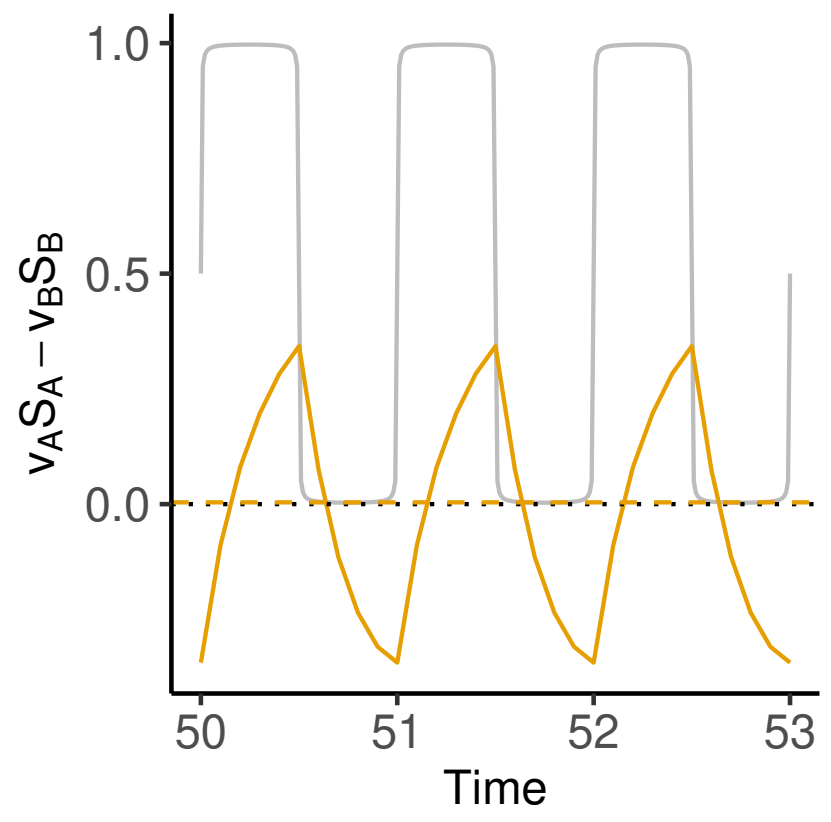

(b) $T=80$

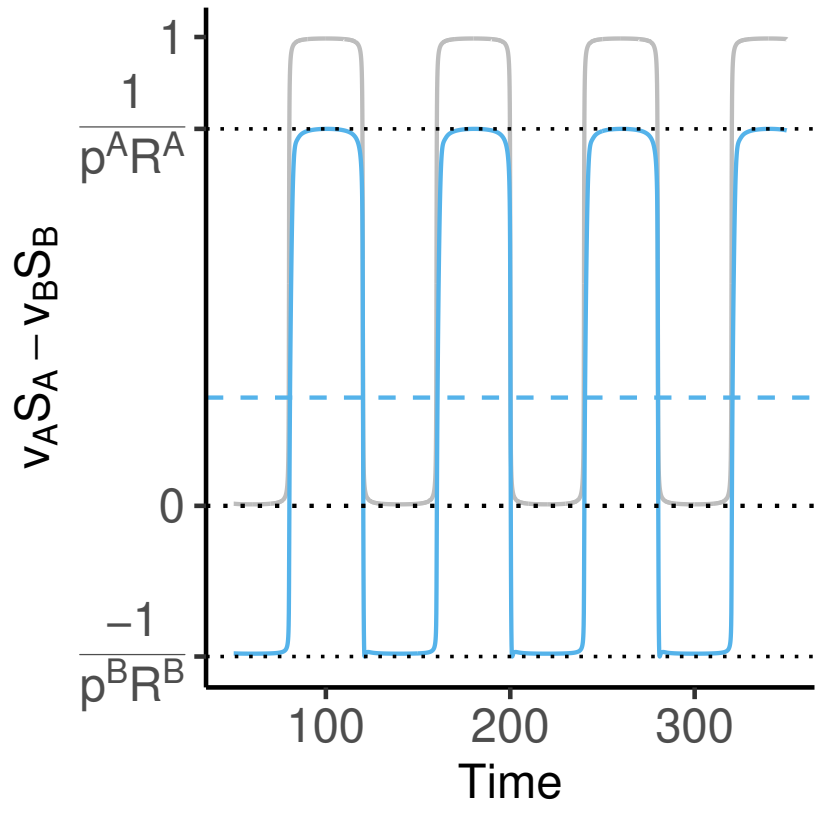

(c)

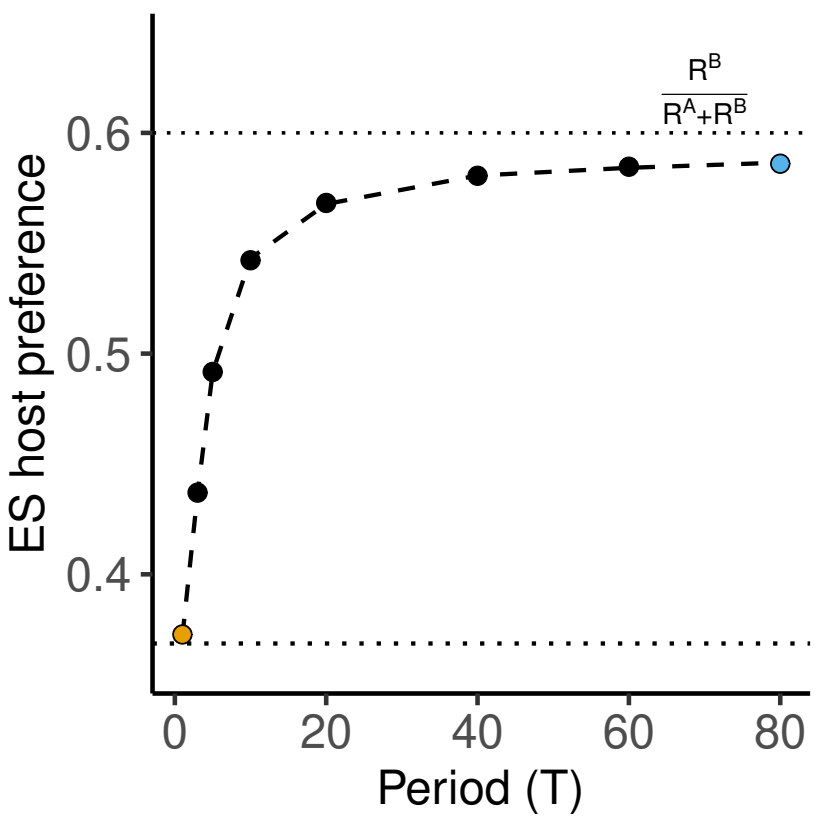

(d)

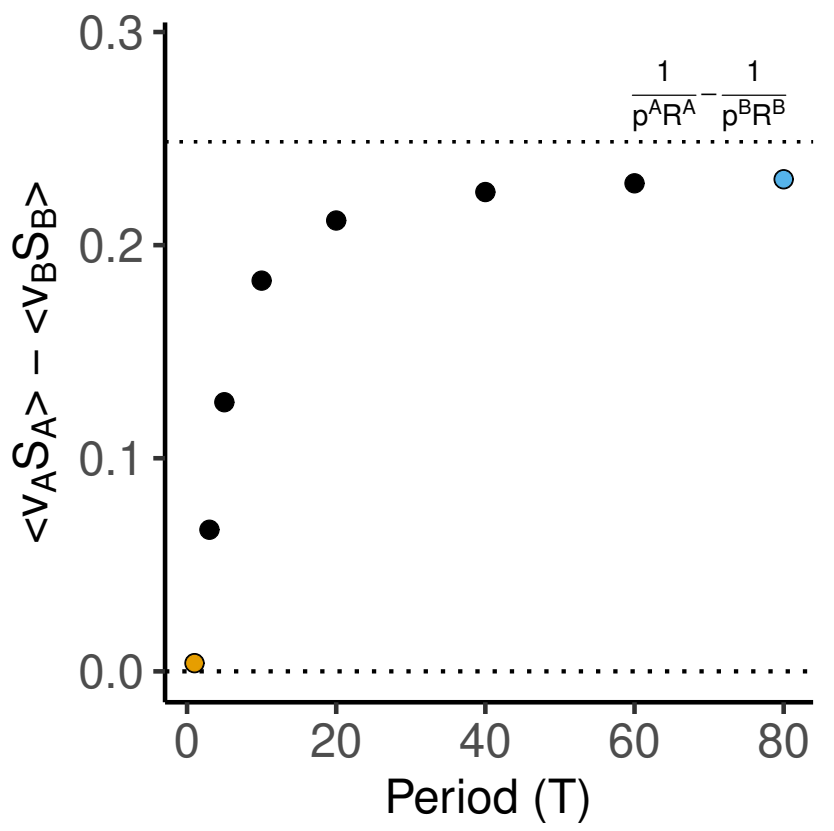

Figure 4: Scenario 2: host preference. (a) Dynamics of $v_{A}(t) S_{A}(t)-v_{B}(t) S_{B}(t)$ for $T=1$ and $z_{w}=z_{e q}$. The dashed line indicates the mean value and the grey line gives the dynamics of $\nu(t)$. (b) Same as (a) but with $T=80$. (c) Predicted ESS as a function of the period of $\nu(t)$ using the reproductive-value-based approach (dots) and the Floquet exponent (dashed line). The dotted line gives the prediction of the corresponding equilibrium model, $z_{e q}=0.368652$. (d) Mean value of the difference in reproductive output $v_{A}(t) S_{A}(t)-v_{B}(t) S_{B}(t)$ as a function of the period for $z_{w}=z_{e q}$. Parameters: $\nu(t)=0.4(1+(2 / \pi) \arctan (\sin (2 \pi t / T) / 0.01)), b=2, d^{A}=d^{B}=1, \beta^{A}=\beta^{B}=\beta=10$, $\alpha^{A}=2, \alpha^{B}=1$. 
This useful result allows us to rewrite the average selection gradient as

$$
\mathcal{S} \approx\left\langle c^{A}\right\rangle \underbrace{\left[\frac{\mathrm{d} \beta^{A}}{\mathrm{~d} z} \frac{d+\alpha^{A}}{\beta^{A}}-\frac{\mathrm{d} \alpha^{A}}{\mathrm{~d} z}\right]}_{\mathcal{S}^{A}}+\left(1-\left\langle c^{A}\right\rangle\right) \underbrace{\left[\frac{\mathrm{d} \beta^{B}}{\mathrm{~d} z} \frac{d+\alpha^{B}}{\beta^{B}}-\frac{\mathrm{d} \alpha^{B}}{\mathrm{~d} z}\right]}_{\mathcal{S}^{B}}
$$

where we have neglected the covariances $\operatorname{Cov}\left(c^{k}, \omega^{k}\right)$ that arise when taking the mean. Extensive numerical simulations show that this approximation fits very well the prediction of a Floquet analysis (Walter \& Lion, 2021).

A full analysis is beyond the scope of this paper, and we refer the reader to the more complete study by Walter \& Lion (2021). Nonetheless, is is interesting to see that the selection gradient takes the form of a weighted sum of the selection gradients in class $A$ and $B$ respectively, exactly as in constant environments. For a constant vaccination coverage, the ES strategy of host exploitation is a weighted mean of the optima in class $A$ and in class $B$, respectively given by the zeros of $\mathcal{S}^{A}$ and $\mathcal{S}^{B}$. For our anti-transmission vaccine $\left(r_{b}>0\right) \mathcal{S}^{A}=\mathcal{S}^{B}$, and therefore vaccination has no effect on the optimum. Equation (28) shows that the same holds true for periodic environment, and this is confirmed by numerical calculations of the Floquet exponents (Walter \& Lion, 2021). For a vaccine that reduces virulence $\left(r_{a}>0\right)$, however, $\mathcal{S}^{A} \neq \mathcal{S}^{B}$, and the position of the ESS is determined by a single variable, which is the mean value, over one period, of the class reproductive value $c^{A}(t)$ in the resident population.

Figure $5 \mathrm{~b}$ shows that the class reproductive value $c^{A}(t)$ closely tracks the environmental fluctuation $\nu(t)$ when $T$ is large, whereas for short periods it quickly fluctuates around its value in a constant environment. An interesting consequence is that, when the period of fluctuations increases, $\left\langle c^{A}\right\rangle$ increases (figure $5 \mathrm{~d}$, which selects for lower virulence compared to a scenario with constant vaccination coverage (figure $5 \mathrm{c}$ ). For large periods, $\left\langle c^{A}\right\rangle$ converges towards $1 / 2$ (the mean of $\nu(t)$ ), which allows the ES virulence to be analytically calculated (Walter \& Lion (2021), appendix S.3). Note that, in the latter figure, the slight quantitative discrepancy between the prediction of equation (28) and the Floquet analysis is due to the fact that we have neglected the covariances $\operatorname{Cov}\left(c^{k}, \omega^{k}\right)$.

\section{Discussion}

We present a new framework to analyse life-history evolution in temporally fluctuating environments, and use it to study the evolution of pathogen traits in three epidemiological scenarios. Our analysis extends previous adaptive dynamics studies, which use the Lyapunov (or Floquet, for periodic environments) exponent of a rare mutant as a measure of invasion fitness (Metz et al., 1992; Geritz et al., 1998; Klausmeier, 2008; Metz, 2008). However, although for unstructured populations an analytical expression of the Floquet exponent is derivable (Donnelly et al., 2013; Cornet et al., 2014; Gandon, 2016; Ferris \& Best, 2018; Pigeault et al., 2018), only numerical solutions are typically proposed for class-structured populations (Ferris \& Best, 2018). Here, we circumvent this problem and derive an analytical expression of the selection gradient on life-history traits, which represents the first-order approximation of invasion fitness for weak selection. We show how it can be used to better understand the impact of environmental fluctuations on the direction of selection and the potential evolutionary endpoints of life-history evolution.

Our approach is based on the analysis of the dynamics of a reproductive-value-weighted frequency of a mutant. This is a recurring idea in evolutionary biology (Fisher, 1930; Taylor \& Frank, 1996; Lehmann \& Rousset, 2014; Lion, 2018a), but the novelty here is that we use a dynamical definition of reproductive value to quantify the fluctuating quality of a class in a periodic environment (see Lion (2018a) for a general discussion on this topic, and Brommer et al. (2000), Caswell (2001), and Bacaër \& Abdurahman (2008) for other approaches). The resulting expression of the selection gradient can then be obtained by weighting the effect, at time $t$, of a mutation on the transition rates from class $j$ to $k$ by the frequency of class $j$ at time $t$ and the individual reproductive value of class $k$ at time $t$. This has two main implications. First, this allows us to quantify selection at time $t$ in terms of the quantity and quality of each class. As in constant environments, the quantity of a class is given 
(a) $T=1$

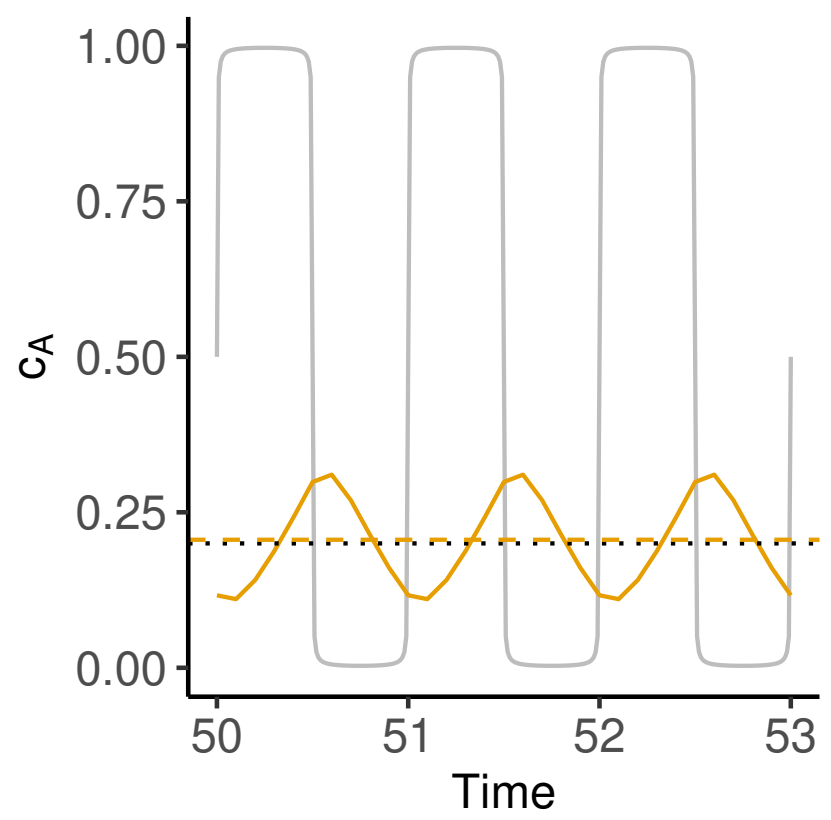

(b) $T=80$

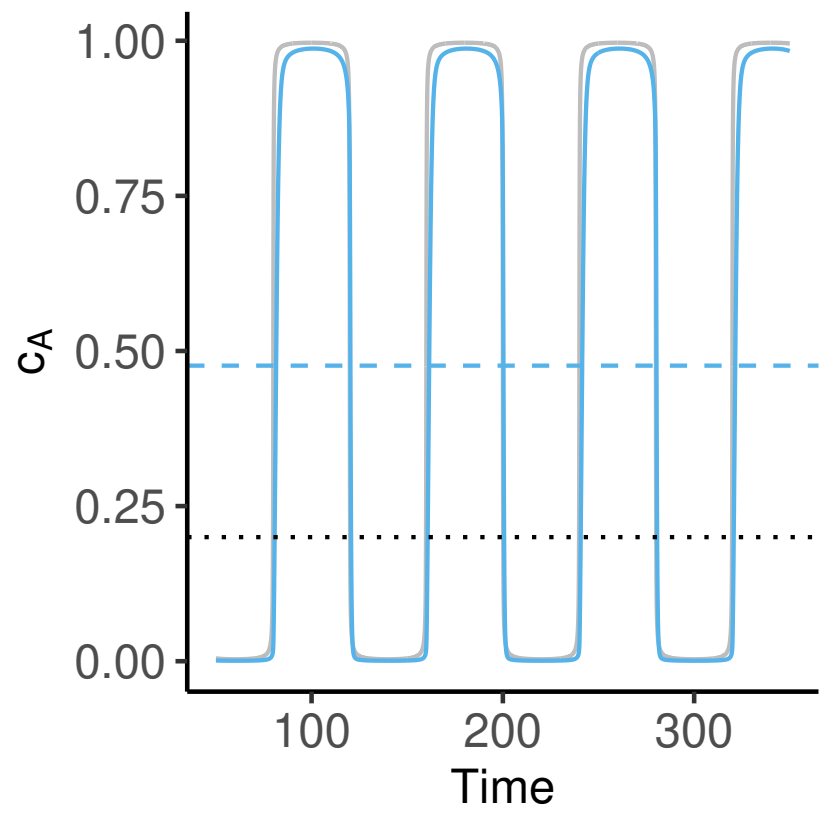

(c)

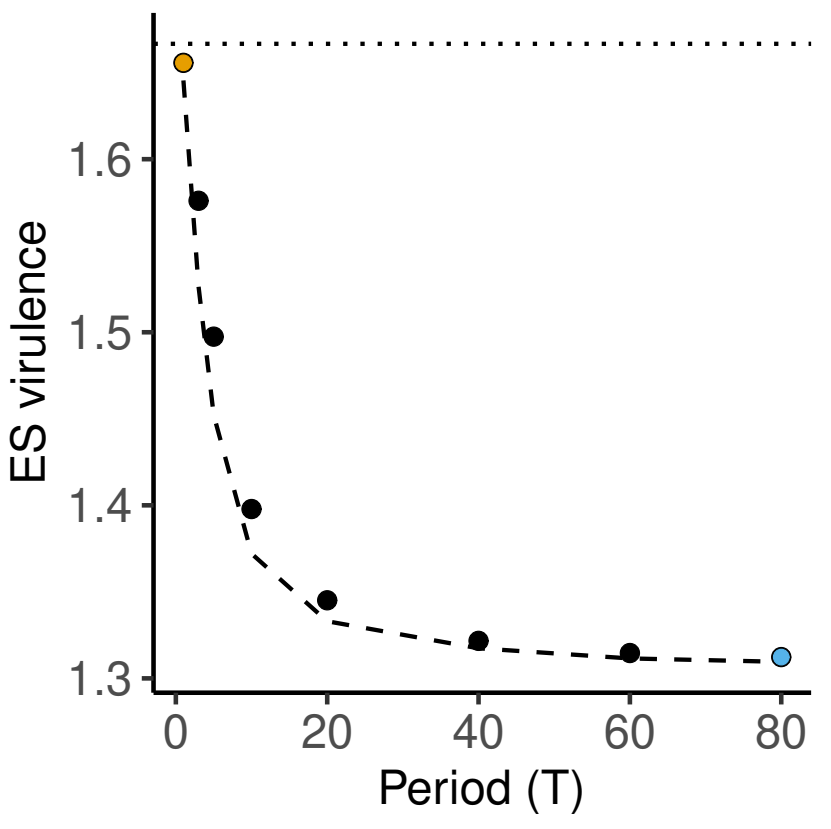

(d)

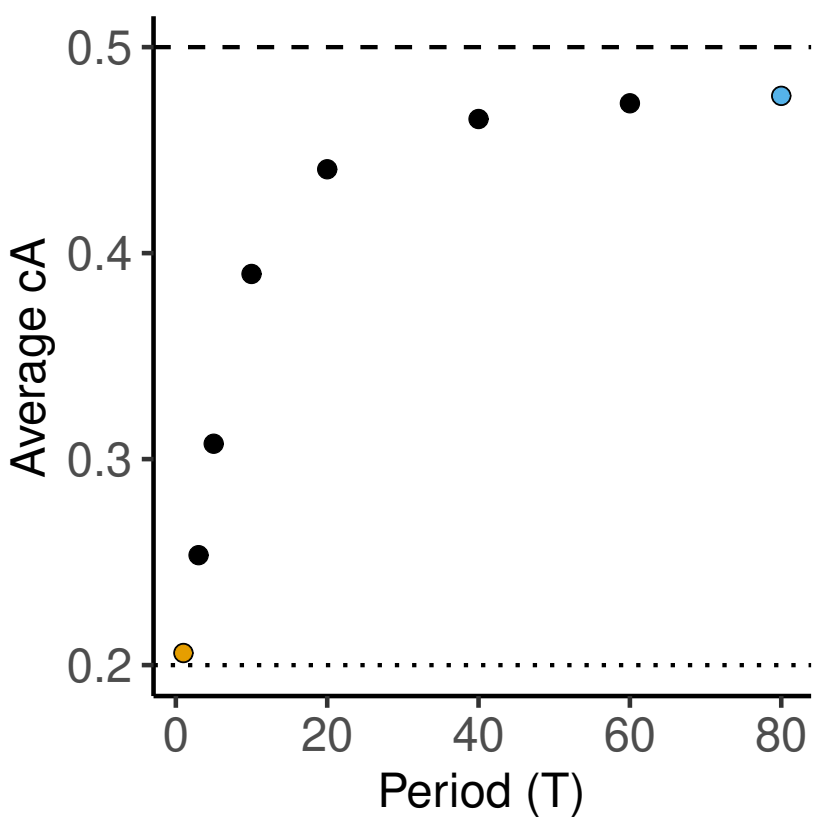

Figure 5: Scenario 3: imperfect vaccines and virulence. (a) Dynamics of the class reproductive value $c^{A}(t)$ for $T=1$ and $z_{w}=z_{e q}$. The dashed line indicates the mean value, the dotted line the value in a constant environment, and the grey line gives the dynamics of $\nu(t)$. (b) Same as (a) but with $T=80$. (c) Predicted ESS as a function of the period of $\nu(t)$ using the reproductive-value-based approach (dots) and the Floquet exponent (dashed line). The dotted line gives the prediction of the corresponding equilibrium model, $z_{e q}=1.667$. (d) Mean value of the class reproductive value $c^{A}(t)$ as a function of the period for $z_{w}=z_{e q}$. Parameters: $\nu(t)=0.5(1+(2 / \pi) \arctan (\sin (2 \pi t / T) / 0.01))$, $b=2, d^{A}=d^{B}=1, \beta^{A}(z)=\beta^{B}(z)=\beta=10 z /(1+z), \alpha^{A}=z, \alpha^{B}=\left(1-r_{a}\right) z, r_{a}=0.8$. 
by the frequency of individuals in that class, and the intrinsic quality of a class is measured by their reproductive value, which gives the relative share of future descendants left by individuals in that class. The only difference is that the quantity and quality of each class are allowed to fluctuate periodically over time. Second, the direction of selection can be obtained by computing the average, over one period of the resident attractor, of the instantaneous selection gradient, and we recover a periodic extension of the "invasion implies fixation" principle (Geritz, 2005; Cai \& Geritz, 2020; Priklopil \& Lehmann, 2020).

It could be argued that, with our approach, the problem of numerically computing a Floquet exponent is replaced by the problem of numerically computing the time-dependent reproductive values and class frequencies. This is of course true if we are simply interested in the quantitative result, but the analytical expression of the selection gradient allows for a qualitative discussion of the selective pressures. As we show in our applications, it is often possible to capture the effect of fluctuations using a single, biologically meaningful quantity. However, as our approach is currently limited to firstorder effects (e.g. convergence stability), the numerical calculation of invasion fitness using Floquet exponents must still be used to evaluate evolutionary stability and determine whether the predicted evolutionary singularities are ESSs or branching points (Appendix B).

Although periodic environmental fluctuations are important in nature, many organisms also experience stochastic environmental fluctuations. An interesting avenue for future work would be to extend our approach to stochastic stationary ecological dynamics. Since the "invasion implies fixation" principle has also been proven for stochastic fluctuations in the environment (Cai \& Geritz, 2020), we think this extension is feasible and would help link our new method with classical theory on the influence of environmental stochasticity on life-history evolution (Frank \& Slatkin, 1990; Sasaki \& Ellner, 1995; Lande et al., 2017). However, this would require a careful definition of the concept of time-dependent reproductive value in stochastic environments.

In our applications, we made two simplifying, but biologically important, assumptions. First, our analysis relies on the classical Adaptive Dynamics assumption that the mutation rate is small. This analysis may predict the evolution of generalist strategies that balance the exploitation of the habitats that fluctuate periodically. Yet, as the period of the fluctuation between different environments increases, one may also expect that new mutations will introduce enough genetic variation to allow the population to adapt to this time-varying environment through the recurrent selection of genotypes specialised to each habitat. Accounting for this type of dynamics requires to track the dynamics of the genetic variance across time (Sasaki \& Dieckmann, 2011; Débarre \& Otto, 2016). Second, we assume that all life-history traits are constant, so that temporal fluctuations only come from periodic variations in birth rates which cause fluctuations in densities. It would be straightforward to consider time-dependent traits (such as a seasonal transmissibility $\beta(t)$ ), but note that this would give rise to additional terms capturing the temporal covariance between the trait and some life-cycle-specific ecological variable (Kamo \& Sasaki, 2005; Koelle et al., 2005; Cornet et al., 2014). Interestingly, allowing traits to vary with the environment provides a way to study the evolution of life-history plasticity. Indeed, plasticity may be a particularly effective way to adapt to a fluctuating environment because it allows the organism to switch between phenotypes specialised to each habitat. This approach has been used to study the evolution of the plasticity of pathogen transmission strategies (Cornet et al., 2014; Gandon, 2016; Pigeault et al., 2018). Our new approach provides a general theoretical framework to extend the study of the evolution of life-history plasticity to class-structured life cycles.

\section{Acknowledgements}

We thank P. Carmona, C. Klausmeier and A. Walter for helpful discussions.

\section{Author contribution}

Both authors contributed equally to this work. 


\section{Funding}

This work was funded by grants ANR-16-CE35-0012 "STEEP" to SL and ANR-17-CE35-0012 "EVOMALWILD" to SG from the Agence Nationale de la Recherche.

\section{References}

Abrams, P., Y. Harada \& H. Matsuda (1993). On the relationship between quantitative genetic and ESS models. Evolution. 47(3): 982-985.

Altizer, S., A. Dobson, P. Hosseini, P. Hudson, M. Pascual \& P. Rohani (2006). Seasonality and the dynamics of infectious diseases. Ecol. Lett. 9(4): 467-484. DOI: $10.1111 /$ j .1461-0248 . 2005 . 00879.x.

Bacaër, N. \& X. Abdurahman (2008). Resonance of the epidemic threshold in a periodic environment. J. math. Biol. 57(5): 649-73. DOI: 10.1007/s00285-008-0183-1.

Barraquand, F. et al. (2017). Moving forward in circles: challenges and opportunities in modelling population cycles. Ecol. Lett. 20: 1074-1092. DOI: 10.1111/ele.12789.

Barton, N. H. \& A. M. Etheridge (2011). The relation between reproductive value and genetic contribution. Genetics. 188: 953-973. DOI: 10.1534/genetics.111.127555.

Berngruber, T. W., S. Lion \& S. Gandon (2015). Spatial structure, transmission modes and the evolution of viral exploitation strategies. PLoS Pathog. 11(4): e1004810. DOI: 10.1371/journal. ppat. 1004810.

Berngruber, T. W., R. Froissart, M. Choisy \& S. Gandon (2013). Evolution of virulence in emerging epidemics. PLoS Pathog. 9: e1003209. DOI: 10.1371/journal.ppat.1003209.

Bonhoeffer, S., R. E. Lenski \& D. Ebert (1996). The curse of the Pharaoh: the evolution of virulence in pathogens with long living propagules. Proc. R. Soc. B. 263(1371): 715-721.

Brommer, J., H. Kokko \& H. Pietiäinen (2000). Reproductive effort and reproductive values in periodic environments. Am. Nat. 155(4): 453-472. DOI: 10.1086/303335.

Cai, Y. \& S. A. H. Geritz (2020). Resident-invader dynamics of similar strategies in fluctuating environments. J. math. Biol. DOI: 10.1007/s00285-020-01532-8.

Caswell, H. (2001). Matrix population models: Construction, analysis, and interpretation. Sinauer Associates.

Cornet, S., A. Nicot, A. Rivero \& S. Gandon (2014). Evolution of plastic transmission strategies in avian malaria. PLoS Pathog. 10(9): e1004308. DOI: 10.1186/s12936-019-2690-5.

Day, T. \& S. Gandon (2006). Insights from Price's equation into evolutionary epidemiology. In: Disease evolution: models, concepts and data analyses. Ed. by Feng, Z., Dieckmann, U. \& Levin, S. 71. DIMACS Series in Discrete Mathematics and Theoretical Computer Science. American Mathematical Society, pp. 23-43.

Débarre, F. \& S. P. Otto (2016). Evolutionary dynamics of a quantitative trait in a finite asexual population. Theor. Pop. Biol. 108: 75-88. DOI: 10.1016/j.tpb.2015.12.002.

Donnelly, R., A. Best, A. White \& M. Boots (2013). Seasonality selects for more acutely virulent parasites when virulence is density dependent. Proc. R. Soc. B. 280(1751): 20122464. DOI: 10 . $1098 / \mathrm{rspb} .2012 .2464$.

Ferris, C. \& A. Best (2018). The evolution of host defence to parasitism in fluctuating environments. J. theor. Biol. 440: 58-65. DOI: 10.1016/j.jtbi.2017.12.006.

Fisher, R. A. (1930). The genetical theory of natural selection. Clarendon Press, Oxford, UK.

Frank, S. A. \& M. Slatkin (1990). Evolution in a variable environment. The American Naturalist. 136(2): 244-260.

Gandon, S. (1998). The curse of the pharaoh hypothesis. Proc. R. Soc. B. 265: 1545-1552. DoI: $10.1098 / \mathrm{rspb} .1998 .0470$.

Gandon, S. (2004). Evolution of multihost parasites. Evolution. 58(3): 455-469. DOI: 10.1111/j.00143820.2004.tb01669.x.

Gandon, S. (2016). Why be temperate: lessons from bacteriophage $\lambda$. Trends in microbiology. 24(5): 356-365. DOI: $10.1016 /$ j.tim.2016.02.008. 
Gandon, S., M. J. Mackinnon, S. Nee \& A. F. Read (2001). Imperfect vaccines and the evolution of pathogen virulence. Nature. 414: 751-756. DOI: 10.1038/414751a.

Gandon, S., M. J. Mackinnon, S. Nee \& A. F. Read (2003). Imperfect vaccination: some epidemiological and evolutionary consequences. Proc. R. Soc. B. 270: 1129-1136. DOI: 10.1098/rspb.2003.2370.

Geritz, S. A. H. (2005). Resident-invader dynamics and the coexistence of similar strategies. J. math. Biol. 50: 67-82. DOI: 10.1007/s00285-004-0280-8.

Geritz, S. A. H., É. Kisdi, G. Meszéna \& J. A. J. Metz (1998). Evolutionarily singular strategies and the adaptive growth and branching of the evolutionary tree. Evol. Ecol. 12: 35-57. DOI: 10. 1023/A: 1006554906681.

Grafen, A. (2015). Biological fitness and the Price equation in class-structured populations. J. theor. Biol. 373: 62-72. DOI: 10.1016/j.jtbi.2015.02.014.

Iwasa, Y., A. Pomiankowski \& S. Nee (1991). The evolution of costly mate preferences: II the "handicap" principle. Evolution. 45: 1431-1442.

Kamo, M. \& A. Sasaki (2005). Evolution toward multi-year periodicity in epidemics. Ecol. Lett. 8(4): 378-385. DOI: $10.1111 / \mathrm{j} .1461-0248.2005 .00734 . \mathrm{x}$.

Klausmeier, C. A. (2008). Floquet theory: a useful tool for understanding nonequilibrium dynamics. Theor. Ecol. 1: 153-161. DOI: 10.1007/s12080-008-0016-2.

Koelle, K., M. Pascual \& M. Yunus (2005). Pathogen adaptation to seasonal forcing and climate change. Proc. R. Soc. B. 272(1566): 971-977. DOI: 10.1098/rspb.2004.3043.

Kremer, C. T. \& C. A. Klausmeier (2013). Coexistence in a variable environment: Eco-evolutionary perspectives. J. theor. Biol. 339: 14-25. DOI: 10.1016/j.jtbi.2013.05.005.

Lande, R., S. Engen \& B.-E. Sæther (2017). Evolution of stochastic demography with life history tradeoffs in density-dependent age-structured populations. Proceedings of the National Academy of Sciences. 114(44): 11582-11590.

Lehmann, L. \& F. Rousset (2014). The genetical theory of social behaviour. Phil. Trans. Roy. Soc. B. 369(1642). DOI: $10.1098 /$ rstb.2013.0357.

Lion, S. (2018a). Class structure, demography and selection: reproductive-value weighting in nonequilibrium, polymorphic populations. Am. Nat. 191(5): 620-637. DOI: https://doi .org/10 . $1101 / 155879$.

Lion, S. (2018b). Theoretical approaches in evolutionary ecology: environmental feedback as a unifying perspective. Am. Nat. 191(1): 21-44. DOI: 10.1086/694865.

Lion, S. \& S. Gandon (2016). Spatial evolutionary epidemiology of spreading epidemics. Proc. R. Soc. B. 283: 20161170. DOI: $10.1098 / \mathrm{rspb} .2016 .1170$.

Martinez, M. E. (2018). The calendar of epidemics: Seasonal cycles of infectious diseases. PLoS pathogens. 14(11): e1007327.

Meszéna, G., M. Gyllenberg, F. J. Jacobs \& J. A. J. Metz (2005). Link between population dynamics and dynamics of Darwinian evolution. Phys. Rev. Lett. 95. DoI: 10 . $1103 /$ PhysRevLett . 95 . 078105.

Metz, J. A. J. (2008). Fitness. In: Encyclopedia of Ecology. Ed. by Jorgensen, S. E. \& Fath, B. D. Academic Press, Oxford, UK, pp. 1599-1612. ISBN: 978-0-08-045405-4. DOI: 10 . 1016 / B978008045405-4.00792-8.

Metz, J. A. J., R. M. Nisbet \& S. A. H. Geritz (1992). How should we define 'fitness' for general ecological scenarios? Trends Ecol. Evol. 7(6): 198-202. DOI: 10.1016/0169-5347 (92) 90073-K.

Osnas, E. E., P. J. Hurtado \& A. P. Dobson (2015). Evolution of pathogen virulence across space during an epidemic. Am. Nat. 185(3): 332-342. DOI: 10.1086/679734.

Otto, S. P. \& T. Day (2007). A Biologist's Guide to Mathematical Modeling in Ecology and Evolution. Princeton University Press, Princeton, NJ.

Pigeault, R., Q. Caudron, A. Nicot, A. Rivero \& S. Gandon (2018). Timing malaria transmission with mosquito fluctuations. Evolution letters. 2(4): 378-389. DOI: 10.1002/ev13.61.

Priklopil, T. \& L. Lehmann (2020). Invasion implies substitution in ecological communities with classstructured populations. Theor. Pop. Biol. 134: 36-52. DOI: 10.1016/j.tpb.2020.04.004.

Rousset, F. (2004). Genetic structure and selection in subdivided populations. Princeton University Press, Princeton, NJ, USA. 
Sasaki, A. \& U. Dieckmann (2011). Oligomorphic dynamics for analyzing the quantitative genetics of adaptive speciation. J. math. Biol. 63(4): 601-35. DOI: 10.1007/s00285-010-0380-6.

Sasaki, A. \& S. Ellner (1995). The evolutionarily stable phenotype distribution in a random environment. Evolution. 49(2): 337-350.

Taylor, P. D. (1990). Allele-frequency change in a class-structured population. Am. Nat. 135(1): 95106. DOI: $10.1086 / 285034$.

Taylor, P. D. \& S. A. Frank (1996). How to make a kin selection model? J. theor. Biol. 180(1): 27-37. DOI: $10.1006 / j$ tbi.1996.0075.

Walter, A. \& S. Lion (2021). Epidemiological and evolutionary consequences of periodicity in treatment coverage. Proc. R. Soc. B. 288: 20203007. DOI: 10.1098/rspb.2020.3007.

\section{Appendix A: Weak-selection approximation of transition rates}

To derive the weak selection approximation of the transition rates, we write $r_{i}^{k j}(\mathbf{E}, t)$ as explicit functions of the phenotypes:

$$
r_{i}^{k j}(\mathbf{E}, t)=r^{k j}\left(z_{i}, \mathbf{E}, t\right)
$$

where $z_{i}$ is the trait of type $i$. Writing $z_{m}=z_{w}+\varepsilon$, for small $\varepsilon$, we use a Taylor expansion to obtain (see e.g. Iwasa et al. (1991), Abrams et al. (1993), Sasaki \& Dieckmann (2011), and Lion (2018b))

$$
r^{k j}\left(z_{m}, \mathbf{E}, t\right)=r^{k j}\left(z_{w}, \mathbf{E}, t\right)+\varepsilon \frac{\partial r^{k j}}{\partial z_{m}}\left(z_{w}, \mathbf{E}, t\right)+O\left(\varepsilon^{2}\right) .
$$

As a result, we have

$$
\begin{aligned}
\bar{r}^{k j}(\mathbf{E}, t) & =r^{k j}\left(z_{w}, \mathbf{E}, t\right)+O(\varepsilon) \\
r_{m}^{k j}(\mathbf{E}, t)-r_{w}^{k j}(\mathbf{E}, t) & =\varepsilon \frac{\partial r^{k j}}{\partial z_{m}}\left(z_{w}, \mathbf{E}, t\right)+O\left(\varepsilon^{2}\right)
\end{aligned}
$$

which implies that the dynamics of the class frequencies and individual reproductive values (as well as extrinsic environmental variables) are $O(1)$ and functions of $r_{w}^{k j}(\mathbf{E}, t)$ only, while the dynamics of $\tilde{f}_{m}$ are $O(\varepsilon)$. This leads to a separation of time scales, with the class frequencies and individual reproductive values being fast variables and the weighted mutant frequency $\tilde{f}_{m}$ being a slow variable. Importantly, the unweighted average, $f_{m}$, is not a slow variable because its dynamics (3) depends on $O(1)$ terms (see also Priklopil \& Lehmann (2020)).

\section{Appendix B: The Floquet approach for a rare mutant}

The classical Adaptive Dynamics approach is based on the assumption that the mutant is rare and on the derivation of invasion fitness as the Lyapunov exponent of the mutant on the resident attractor (Metz et al., 1992; Geritz et al., 1998; Metz, 2008). For periodic attractors, this amounts to calculating the so-called Floquet exponent of the mutant invasion dynamics (Klausmeier, 2008). Unfortunately, Floquet exponents typically have to be numerically calculated, and only in specific cases can an analytical expression be derived. For instance, when the population has only one class $(K=1)$, it is generally straightforward to derive an analytical expression for the invasion fitness of the mutant by integrating its per-capita growth rate over one period (see e.g. Donnelly et al. (2013) and Ferris \& Best (2018)).

The general procedure to calculate the invasion fitness of a rare mutant is to evaluate the mutant dynamics on the resident attractor. This leads to a matrix $\mathbf{R}_{m}\left(\mathbf{E}_{w}, t\right)$, where $\mathbf{E}_{w}$ denotes the environment on the resident attractor. One then numerically integrates the matrix differential equation

$$
\frac{\mathrm{d} \mathbf{X}}{\mathrm{d} t}=\mathbf{R}_{m}\left(\mathbf{E}_{w}, t\right) \mathbf{X}
$$


over one period (from $t=t_{0}$ to $t=t_{0}+T$ ) from the initial condition $\mathbf{X}\left(t_{0}\right)=\mathbf{I}$, the identity matrix. The eigenvalues of $\mathbf{X}\left(t_{0}+T\right)$ are called the Floquet multipliers, and the invasion fitness can then be expressed as the dominant Floquet exponent

$$
\lambda\left(z_{m}, z_{w}\right)=\frac{1}{T} \ln \mu
$$

where $\mu$ is the dominant Floquet multiplier. This numerical procedure can be repeated for any combination of the mutant and resident traits, which makes it possible to develop the full toolbox of Adaptive Dynamics to investigate the convergence and evolutionarily stability of the singularities. Although well established in theory, this method is very rarely encountered in practice in the literature, because very few studies have actually analysed long-term life-history evolution in periodic environments for class-structured populations (but see e.g. Ferris \& Best (2018)). 


\section{Appendix S: Supplementary Online Material}

\section{S.1 Scenario 1: Curse of the Pharaoh}

Figure S.1a shows that fluctuations in the production of susceptible hosts (top panel) cause fluctuations in the quantity and quality of classes $A$ and $B$ (middle and bottom panels). By averaging over these fast fluctuations, it is possible to track the frequency $f_{m}(t)$ of the mutant. Figure S.1b shows that our reproductive-value-weighted selection gradient $(\langle\mathcal{S}\rangle$, equation (13); dashed line) gives a very accurate prediction for the dynamics of the mutant frequency $f_{m}(t)$ (grey) on the slow time scale (top panel). When one zooms in, we recover the fast fluctuations of the instantaneous selection gradient $\mathcal{S}(t)$ (bottom panel). Note that the direction of selection is well predicted by the sign of $\mathcal{S}(t)$.

For the life cycle of Scenario 1, we can use equation (6) to derive the following dynamics of class frequencies:

$$
\begin{aligned}
& \frac{\mathrm{d} f^{A}}{\mathrm{~d} t}=\beta^{B} S(t) f^{B}(t)-\left(d^{A}+\alpha^{A}\right) f^{A}(t)-\bar{r} f^{A}(t) \\
& \frac{\mathrm{d} f^{B}}{\mathrm{~d} t}=\beta^{A} f^{A}(t)-d^{B} f^{B}(t)-\bar{r} f^{B}(t)
\end{aligned}
$$

together with the dynamical equation for $S(t)$. There is no analytical solution in the general case, but numerical integration allows us to investigate the dynamics of the class frequencies. Note that the frequency of free-living pathogens (class $B$ ) will always tend to lag behind the class $A$ of infected hosts because these free-living pathogens are produced from class $A$.

In the absence of fluctuations $(\zeta=0)$, the system reaches an equilibrium, which can be calculated by setting $\mathrm{d} f^{k} / \mathrm{d} t$ and $\bar{r}$ to zero. We thus obtain

$$
\frac{f^{A *}}{f^{B *}}=\frac{d^{B}}{\beta^{A}} \quad \text { and } \quad S^{*}=\frac{d^{A}+\alpha^{A}}{\beta^{B}} \frac{d^{B}}{\beta^{A}} .
$$

In the presence of fluctuations $(\zeta>0)$, an explicit expression of $f^{A}(t)$ on the periodic attractor is beyond our reach, but, because

$$
\left\langle\frac{\mathrm{d} \ln \left(f^{k}\right)}{\mathrm{d} t}\right\rangle=\langle\bar{r}\rangle=0
$$

we can show that

$$
\left\langle\frac{f^{A}}{f^{B}}\right\rangle=\frac{d^{B}}{\beta^{A}} \quad \text { and } \quad\left\langle\frac{S f^{B}}{f^{A}}\right\rangle=\frac{d^{A}+\alpha^{A}}{\beta^{B}} .
$$

Hence, the ratio of class frequencies, $f^{A}(t) / f^{B}(t)$, fluctuates around an average value $d^{B} / \beta^{A}$, which corresponds to the ratio of class frequencies in the absence of fluctuations. In particular, this implies that, when $d^{B} \rightarrow 0$ (i.e. when propagules live very long), $f^{A}(t) \rightarrow 0$. However, this information cannot be used to further simplify the selection gradient (17).

In contrast, the dynamics of reproductive values yield a very useful expression for the average of the ratio of individual reproductive values, $v^{B}(t) / v^{A}(t)$, on the periodic attractor. From equation (8) we obtain

$$
\begin{aligned}
& \frac{\mathrm{d} v^{A}}{\mathrm{~d} t}=-\beta^{A} v^{B}(t)+\left(d^{A}+\alpha^{A}\right) v^{A}(t)+\bar{r} v^{A}(t) \\
& \frac{\mathrm{d} v^{B}}{\mathrm{~d} t}=-\beta^{B} S(t) v^{A}(t)+d^{B} v^{B}(t)+\bar{r} v^{B}(t)
\end{aligned}
$$

and, using the fact that $\left\langle\mathrm{d} \ln \left(v^{k}\right) / \mathrm{d} t\right\rangle=0$ on the periodic attractor, we obtain

$$
\left\langle\frac{v^{B}}{v^{A}}\right\rangle=\frac{d+\alpha^{A}}{\beta^{A}} \quad \text { and } \quad\left\langle\frac{v^{A} S}{v^{B}}\right\rangle=\frac{d^{B}}{\beta^{B}} .
$$

It is straightforward to check that the right-hand sides correspond to the equilibrium values in the absence of fluctuations. 
This result suggests that it may be useful to rewrite equation (17) to reveal the selection gradient in a constant environment. We thus write:

$$
\mathcal{S}(t)=c^{A}(t)\left(\frac{v^{B}(t)}{v^{A}(t)} \frac{\mathrm{d} \beta^{A}}{\mathrm{~d} z}-\frac{\mathrm{d} \alpha^{A}}{\mathrm{~d} z}\right)
$$

where $c^{A}(t)=v^{A}(t) f^{A}(t)$ is the class reproductive value at time $t$ (Taylor, 1990; Rousset, 2004; Lion, 2018a). Averaging over the period then gives equation (20) in the main text:

$$
\langle\mathcal{S}\rangle=\left\langle c^{A}\right\rangle \mathcal{S}_{c}+\frac{\mathrm{d} \beta^{A}}{\mathrm{~d} z} \operatorname{Cov}\left(c^{A}(t), \frac{v^{B}(t)}{v^{A}(t)}\right) .
$$

In the absence of fluctuations, or when the covariance is zero, the ESS is predicted from $\mathcal{S}_{c}=0$, which takes the form of a simple marginal value theorem:

$$
\frac{\frac{\mathrm{d} \beta^{A}}{\mathrm{~d} z}}{\frac{\mathrm{d} \alpha^{A}}{\mathrm{~d} z}}=\frac{\beta^{A}}{d+\alpha^{A}}
$$

In other words, the ESS maximises the ratio $\beta^{A} /\left(d+\alpha^{A}\right)$. Any departure from this prediction is caused by a non-zero covariance between $c^{A}(t)$ and $\frac{v^{B}(t)}{v^{A}(t)}$. We can develop this covariance and use (S.4) to express this covariance as a function of the reproductive outputs through class $A\left(v^{A}(t) f^{A}(t)\right)$ and through class $B\left(v^{B} f^{A}(t)\right)$. We obtain:

$$
\operatorname{Cov}\left(c^{A}(t), \frac{v^{B}(t)}{v^{A}(t)}\right)=\left\langle v^{B} f^{A}\right\rangle-\left\langle v^{A} f^{A}\right\rangle\left\langle\frac{v^{B}}{v^{A}}\right\rangle=\left\langle v^{B} f^{A}\right\rangle-\left\langle v^{A} f^{A}\right\rangle \frac{d^{A}+\alpha^{A}}{\beta^{A}}
$$

so that the sign of this covariance tells us whether the ratio of the average reproductive outputs is greater or smaller than the average of the ratio of reproductive outputs. In other words, the covariance is positive if

$$
\frac{\left\langle v^{B} f^{A}\right\rangle}{\left\langle v^{A} f^{A}\right\rangle}>\left\langle\frac{v^{B} f^{A}}{v^{A} f^{A}}\right\rangle=\left\langle\frac{v^{B}}{v^{A}}\right\rangle=\frac{d^{A}+\alpha^{A}}{\beta^{A}}
$$

and negative otherwise.

As a final remark on this model, we note that, as in earlier studies (Bonhoeffer et al., 1996; Day \& Gandon, 2006), we have neglected the decrease in propagule density due to the infection of new hosts. This effect can be captured by adding a $-\beta^{B} S(t) I^{B}(t)$ term to the dynamics of $I^{B}$, but this has no qualitative impact on our results.

\section{S.2 Scenario 2: host preference}

Figure S.2a shows that, with our choice of periodic fluctuations in $\nu(t)$ (top panel), the system alternates between periods of high densities of susceptible $A$ hosts and intermediate densities of susceptible $B$ hosts (middle panel). When one host is present, the other is either absent or present at low densities. The resulting dynamics of reproductive values (bottom panel) show that the qualities of $A$ and $B$ hosts fluctuate over time, but that $B$ hosts are always more valuable than $A$ hosts. However, some degree of preference for $A$ hosts $(z>0)$ may evolve because the densities of susceptible hosts are not equal. This can be shown both for constant and periodic environments.

In the constant case, it is easy to show that, in the resident population at equilibrium

$$
\frac{v^{A *}}{v^{B *}}=\frac{R^{A}}{R^{B}}
$$

where $R^{k}=\beta^{k} /\left(d+\alpha^{k}\right)$ is the basic reproductive ratio in a fully suceptible populations with only $k$ hosts present. Thus at the ESS the following condition must be satisfied:

$$
R^{A} S^{A *}=R^{B} S^{B *}
$$


(a) Fast variables

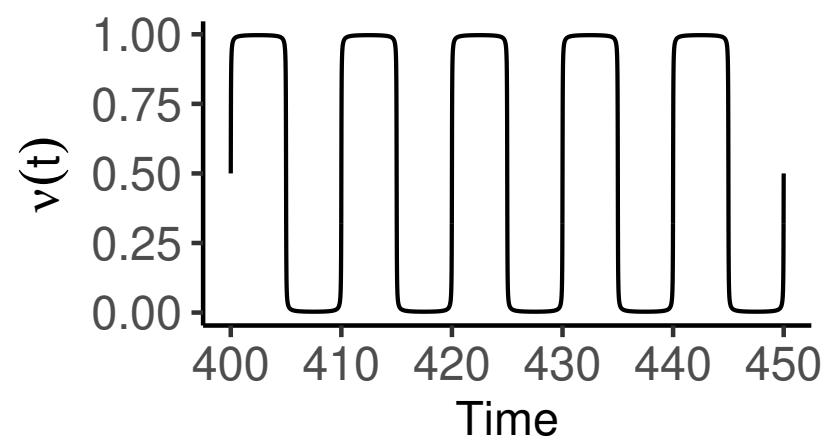

(b) Slow variable

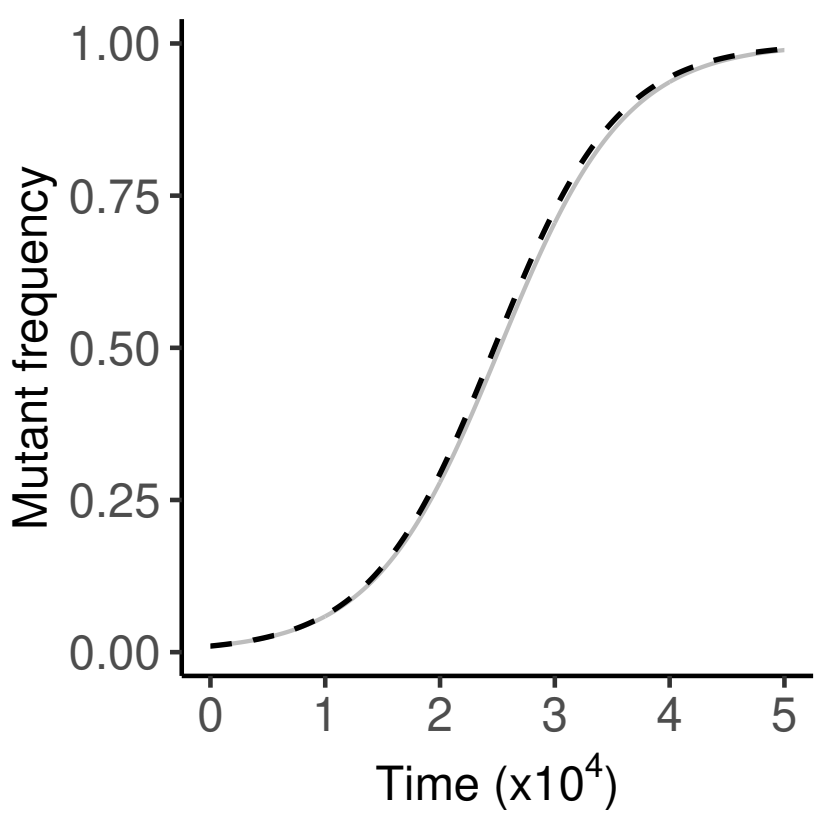

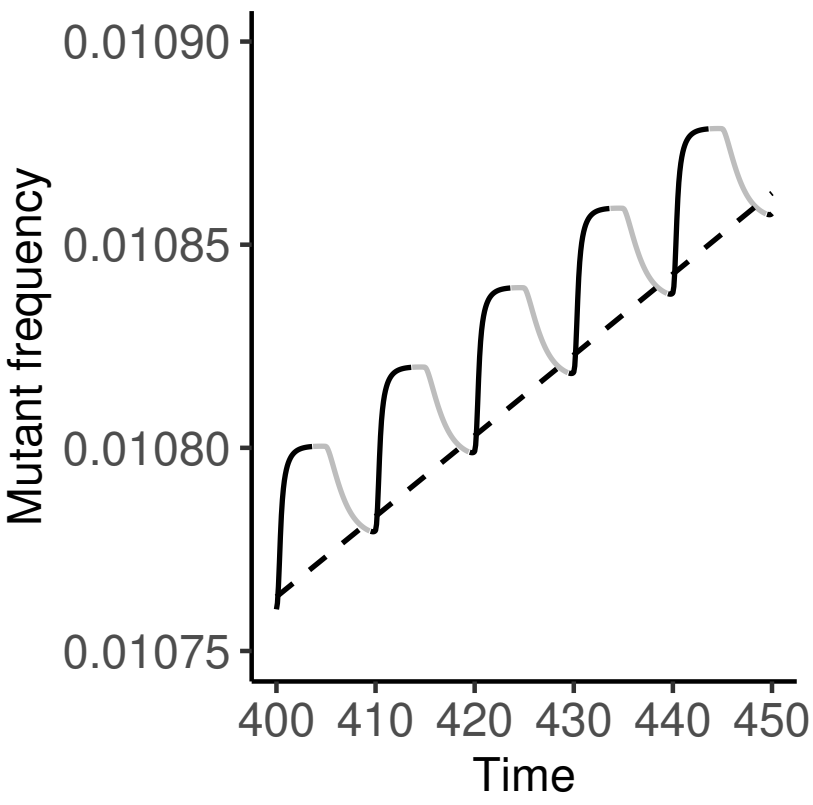

Figure S.1: Scenario 1: The Curse of Pharaoh. (a) Ecological dynamics on the fast time scale. Top: periodic probability of production of susceptible hosts, $\nu(t)$. Middle: dynamics of host densities (blue: susceptible hosts, red: infected hosts (solid line: $A$ class, dashed line: $B$ class (propagules)). Bottom: dynamics of individual reproductive values for pathogens in $A$ (solid) and $B$ (dashed) hosts. (b) Slow-time dynamics of the frequency of a mutant $f_{m}(t)$ (grey) compared to the prediction using the average selection gradient (dashed line). The lower panel is a zoom showing the oscillations of the mutant frequency on the fast time scale. The direction of selection is well predicted by the sign of the instantaneous selection gradient $\mathcal{S}(t)$ (black: positive, grey: negative). Parameters: $\nu(t)=$ $0.5(1+(2 / \pi) \arctan (\sin (2 \pi t / T) / 0.01)), b=8, d=d^{A}=d^{B}=1, \beta^{A}(z)=\beta_{0} z /(1+z), \beta^{B}=\beta_{0}=10$, $\alpha^{A}(z)=z, z_{w}=1, z_{m}=z_{w}+0.005, T=10$. The value of $z_{w}$ is the ESS value in a constant environment with $\nu=0.5$. 
which can be numerically solved to yield an intermediate ES value $z_{e q}^{*}$.

In the periodic case, the selection gradient vanishes when

$$
\left\langle v^{A} S^{A}\right\rangle=\left\langle v^{B} S^{B}\right\rangle
$$

To see how periodic fluctuations can affect the ES preference strategy, we now fix the resident trait at $z_{w}=z_{e q}^{*}$, and track the frequency of a mutant with a slightly increased preference for $A$ hosts $\left(z_{m}=z_{w}+0.001\right)$. In the absence of fluctuations, this mutant should be counter-selected. In figure $\mathrm{S} .2 \mathrm{~b}$, we show that the dynamics of the mutant frequency in the full eco-evolutionary model (solid grey line) is very well predicted, on the slow time scale, by equation (12) with $\langle\mathcal{S}\rangle=\beta\left(\left\langle v^{A} S^{A}\right\rangle-\left\langle v^{B} S^{B}\right\rangle\right)$ (dashed line), as expected from our general mathematical analysis. Biologically, this means that, although at all times pathogens in $B$ hosts have a higher individual reproductive value than pathogens in $A$ hosts (figure S.2a, bottom panel), a mutant with increased preference for $A$ hosts can still be favoured if the fluctuations in the densities of susceptible hosts tilt the balance in the right direction. In the main text, we show that the deviation from the ESS in the constant environment, $z_{\text {eq }}^{*}$, becomes larger as the period, $T$, increases.

In the limit of large periods, for a function $\nu$ that approaches a step function with minimum 0 , maximum 1 and mean 1/2, it is possible to obtain an analytical expression of the ESS. We note that, for large periods, the system approximately behaves as an alternance of single-class equilibria. When only $\mathrm{A}$ hosts are present, we have $S^{B}=0, v^{A}=1$ and $S^{A}=1 /\left(p^{A} R^{A}\right)$. When only B hosts are present, we have $S^{A}=0, v^{B}=1$ and $S^{B}=1 /\left(p^{B} R^{B}\right)$. Thus, we have $v^{A} S^{A}-v^{B} S^{B}=$ $(1 / 2)\left[1 /\left(p^{A} R^{A}\right)-1 /\left(p^{B} R^{B}\right)\right]$, so that, for $p^{A}=z=1-p^{B}$ we obtain

$$
z^{*}=\frac{R^{B}}{R^{A}+R^{B}}
$$

which is the upper limit in figure 4c.

Note that the simulations are performed without any cost of preference, so that the singularity is actually degenerate (i.e. the second isocline of the PIP is vertical near the ESS). However, adding a small constant cost $c=1.5(z-1 / 2)^{2}$ to the death rates of infected hosts in both classes is enough to make the singularity evolutionarily stable. This only adds a small negative term to the selection gradient that is independent of fluctuations, and has no qualitative impact on our results.

\section{S.3 Scenario 3: imperfect vaccines}

As for scenario 2, figure S.3 shows the dynamics on both the fast, ecological time scale and the slow, evolutionary time scale for a given set of parameter values (chosen such that the trait is at the ESS value in the absence of fluctuations). Again, figure S.3b shows that the averaged selection gradient using reproductive values accurately predicts the dynamics of the mutant frequency $f_{m}(t)$.

The dynamics of the individual reproductive values are given by the system:

$$
\begin{aligned}
& \frac{\mathrm{d} v^{A}}{\mathrm{~d} t}=\beta^{A}\left(v^{A} p^{A} S^{A}+v^{B} p^{B} S^{B}\right)-\left(d+\alpha^{A}+\bar{r}\right) v^{A}, \\
& \frac{\mathrm{d} v^{B}}{\mathrm{~d} t}=\beta^{B}\left(v^{A} p^{A} S^{A}+v^{B} p^{B} S^{B}\right)-\left(d+\alpha^{B}+\bar{r}\right) v^{B} .
\end{aligned}
$$

Because on the periodic attractor, we have

$$
\left\langle\frac{\mathrm{d} \ln \left(v^{k}\right)}{\mathrm{d} t}\right\rangle=0 \quad \text { and } \quad\langle\bar{r}\rangle=0,
$$

it follows that

$$
\left\langle\frac{v^{A} p^{A} S^{A}+v^{B} p^{B} S^{B}}{v^{k}}\right\rangle=\frac{d+\alpha^{k}}{\beta^{k}}=\frac{1}{R^{k}}
$$


(a) Fast variables
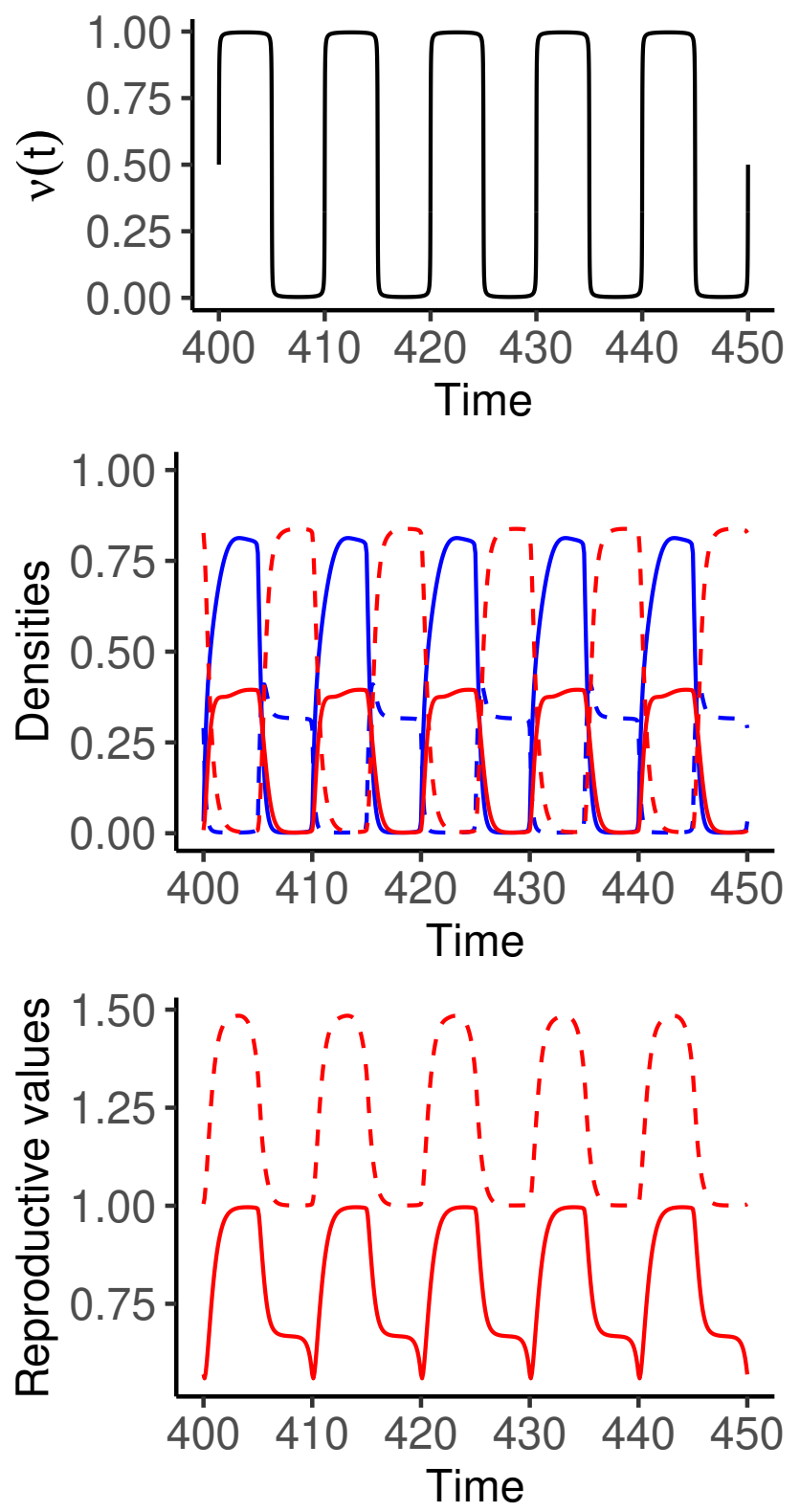

(b) Slow variable
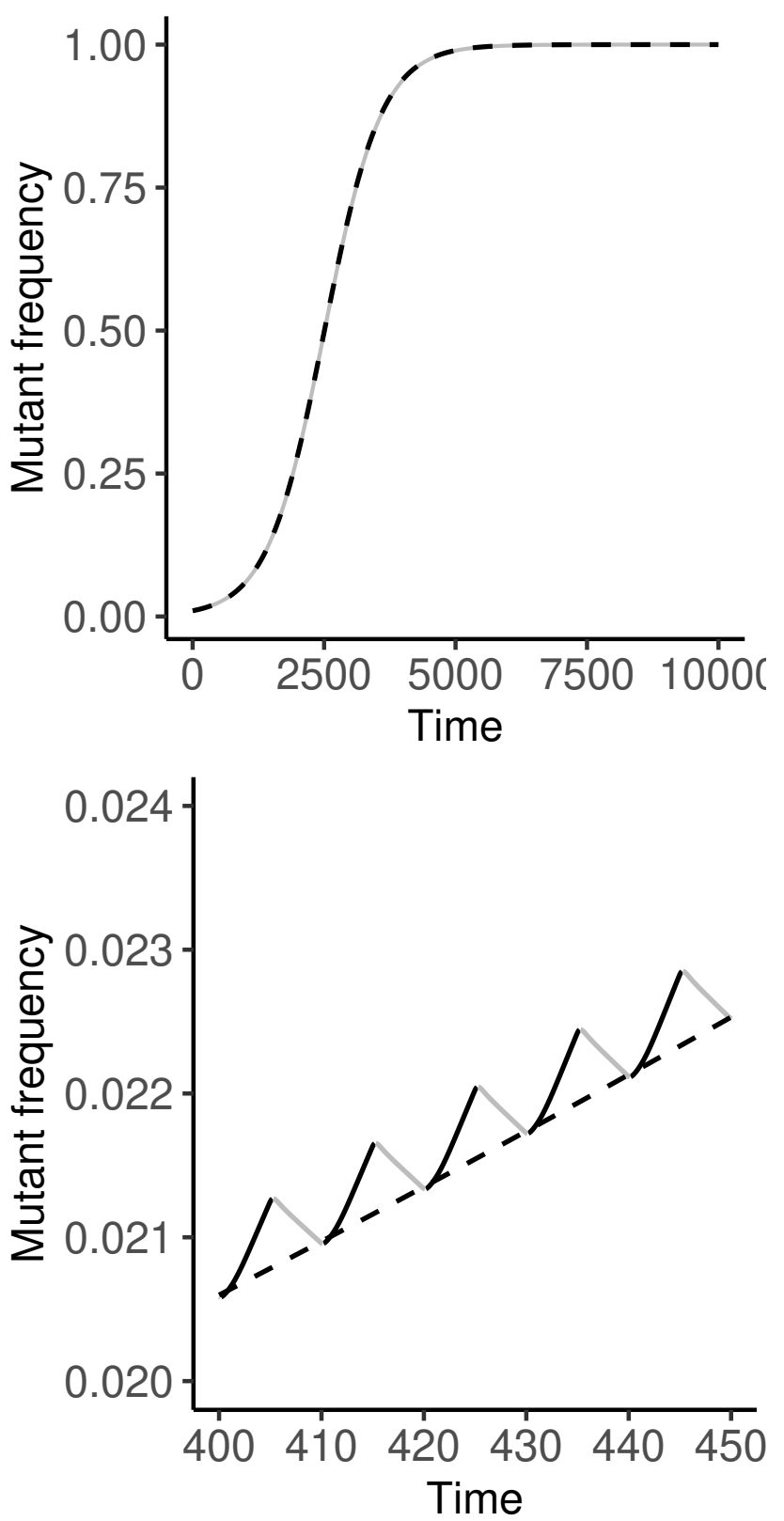

Figure S.2: Scenario 2: host preference. (a) Ecological dynamics on the fast time scale. Top: periodic probability of production of $A$ susceptible hosts, $\nu(t)$. Middle: dynamics of host densities (blue: susceptible hosts, red: infected hosts, solid lines: $A$ hosts, dashed lines: $B$ hosts). Bottom: dynamics of individual reproductive values for pathogens in $A$ (solid) and $B$ (dashed) hosts. (b) Slow-time dynamics of the frequency of a mutant $f_{m}(t)$ (grey) compared to the prediction using the average selection gradient (dashed line). The lower panel is a zoom showing the oscillations of the mutant frequency on the fast time scale. The direction of selection is well predicted by the sign of $v^{A}(t) S^{A}(t)-v^{B}(t) S^{B}(t)$ (black: positive, grey: negative). Parameters: $\nu(t)=0.5(1+(2 / \pi) \arctan (\sin (2 \pi t / T) / 0.01)), b=2$, $d^{A}=d^{B}=1, \beta^{A}=\beta^{B}=\beta=10, \alpha^{A}=2, \alpha^{B}=1, z_{w}=0.368652, z_{m}=z_{w}+0.001, T=10$. The value of $z_{w}$ is the ESS value in a constant environment $\nu=0.5$. 
In figure S.4 (bottom panel), we show that the mean of the ratio $\omega^{k}=\left(v^{A} p^{A} S^{A}+v^{B} p^{B} S^{B}\right) / v^{k}$ is indeed equal to $1 / R^{k}$. Furthermore, as the period becomes large, $\omega^{k}$ is nearly always equal to $1 / R^{k}$ except for brief deviations when the environment changes. On the other hand, the middle panel shows that the mean of the reproductive values $\left\langle v^{k}\right\rangle$ are close to their values in a constant environment $v_{e q}^{k}$ for short periods, but as the period increases, so does the difference between $\left\langle v^{k}\right\rangle$ and $v_{e q}^{k}$.

In the limit of large periods, $c^{A}(t)$ converges towards $\nu(t)$. This can be seen by noting that, for large periods, the model essentially behaves as a succession of single-class equilibria. Half the time, only $A$ hosts are present, so that $c^{A}=1$. The rest of the time, there are only $B$ hosts and $c^{A}=0$. Hence the mean value of the class reproductive value converges towards $1 / 2$, and the selection gradient simplies to $\langle\mathcal{S}\rangle=\left(\mathcal{S}^{A}+\mathcal{S}^{B}\right) / 2$. With a trade-off $\alpha^{A}=z, \alpha^{B}=\left(1-r_{a}\right) z$ and $\beta^{B}=\beta^{A}=\beta_{0} z /(1+z)$, the ES virulence for large periods has the very simple expression:

$$
z^{*}=\frac{1}{\sqrt{1-r_{a} / 2}}
$$

(see also Walter \& Lion (2021) for a slightly more general result).

Finally, we note that the dynamics of the individual reproductive values can be used to show that, for a vaccine that linearly reduces transmission (i.e. $r_{a}=0$ and $r_{b}>0$ ), we have

$$
\frac{\mathrm{d}\left[\left(1-r_{b}\right) v^{A}-v^{B}\right]}{\mathrm{d} t}=0
$$

which means that, although the reproductive values fluctuate due to the dynamics of the host densities, their ratio $v^{A}(t) / v^{B}(t)$ remains constant and equal to $1 /\left(1-r_{b}\right)$ at all times. This can be confirmed numerically (results not shown). 
(a) Fast time scale
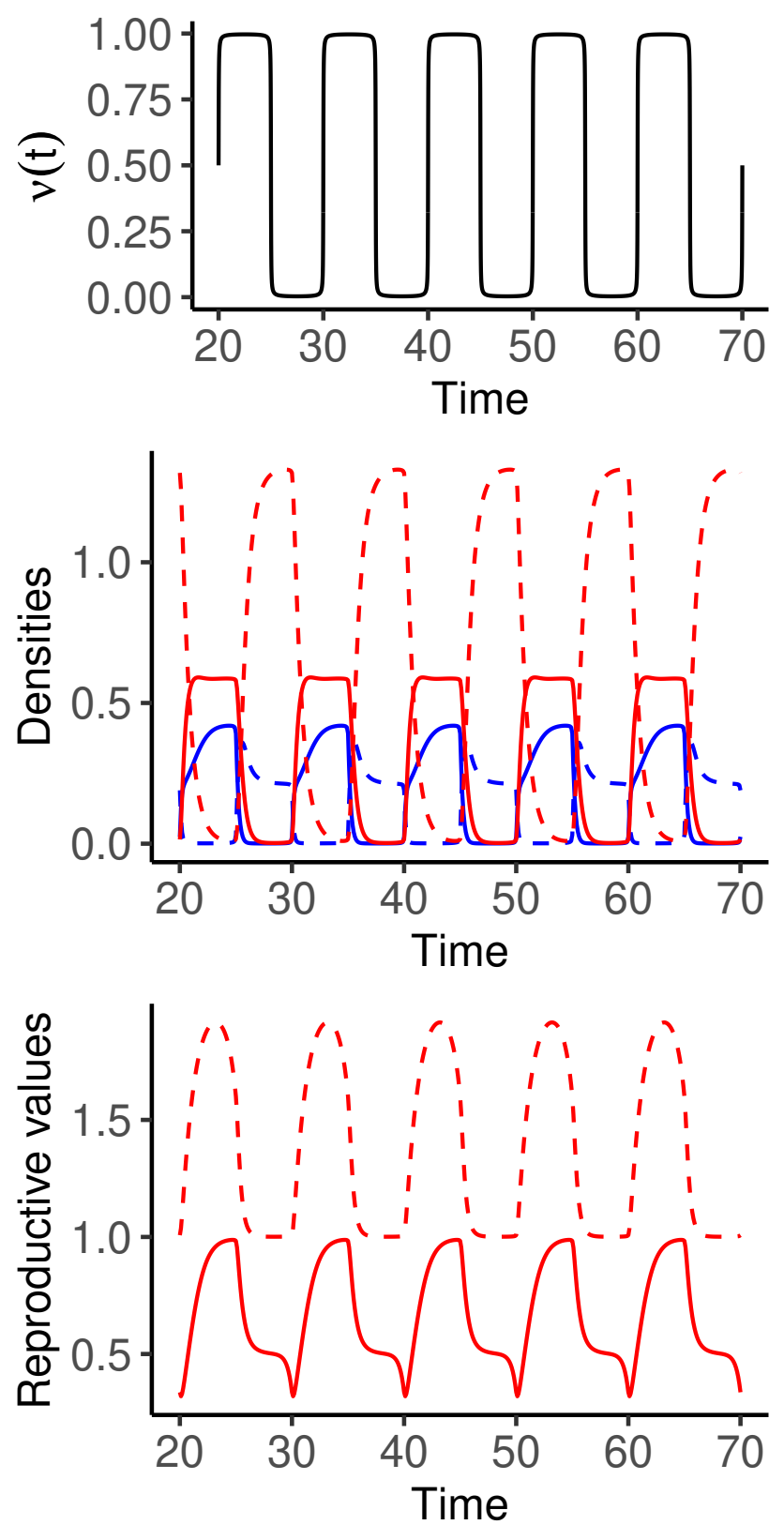

(b) Slow time scale

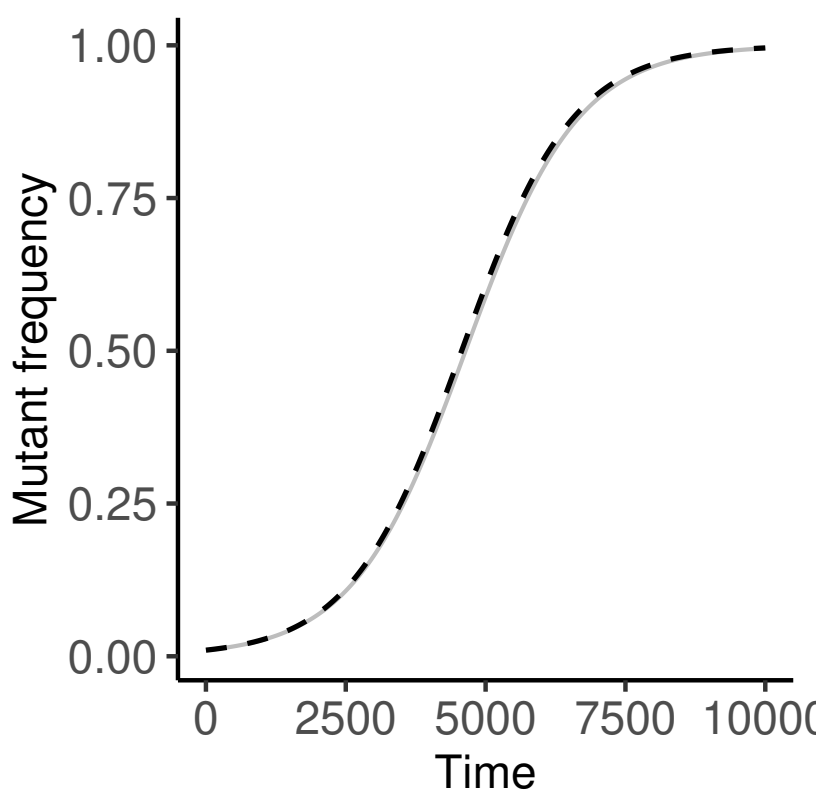

Figure S.3: Scenario 3: imperfect vaccine and virulence. (a) Ecological dynamics on the fast time scale. Top: periodic probability of production of $A$ susceptible hosts, $\nu(t)$. Middle: dynamics of host densities (blue: susceptible hosts, red: infected hosts, solid lines: $A$ hosts, dashed lines: $B$ hosts). Bottom: dynamics of individual reproductive values for pathogens in $A$ (solid) and $B$ (dashed) hosts. (b) Slow-time dynamics of the frequency of a mutant $f_{m}(t)$ (grey) compared to the prediction using the average selection gradient (dashed line). Parameters: $\nu(t)=0.5(1+(2 / \pi) \arctan (\sin (2 \pi t / T) / 0.01))$, $b=2, d^{A}=d^{B}=1, \beta^{A}(z)=\beta^{B}(z)=\beta=10 z /(1+z), \alpha^{A}=z, \alpha^{B}=\left(1-r_{a}\right) z, r_{a}=0.8, z_{w}=1.667$, $z_{m}=z_{w}-0.01, T=10$. The value of $z_{w}$ is the ESS value in a constant environment $\nu=0.5$. 
(a) $T=1$
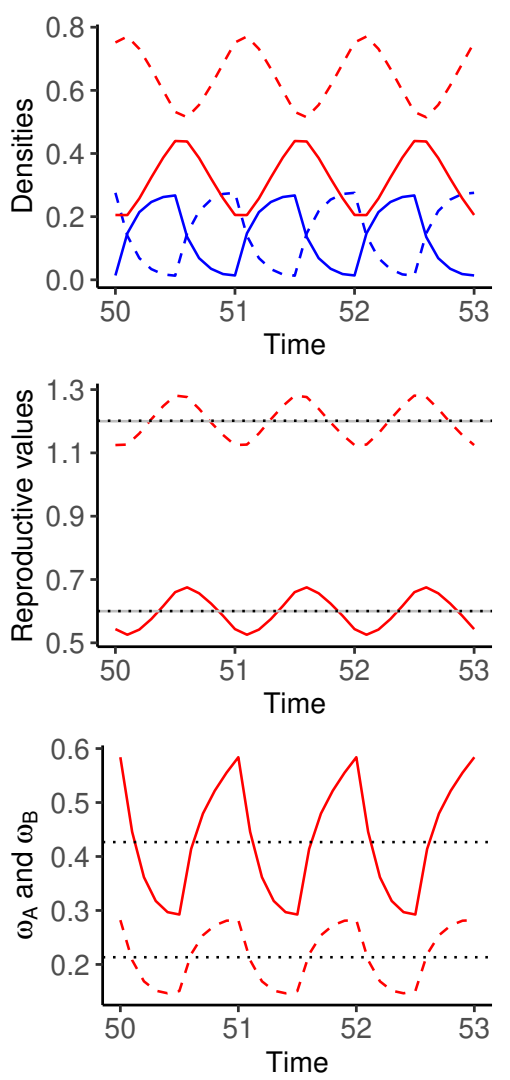

(b) $T=10$
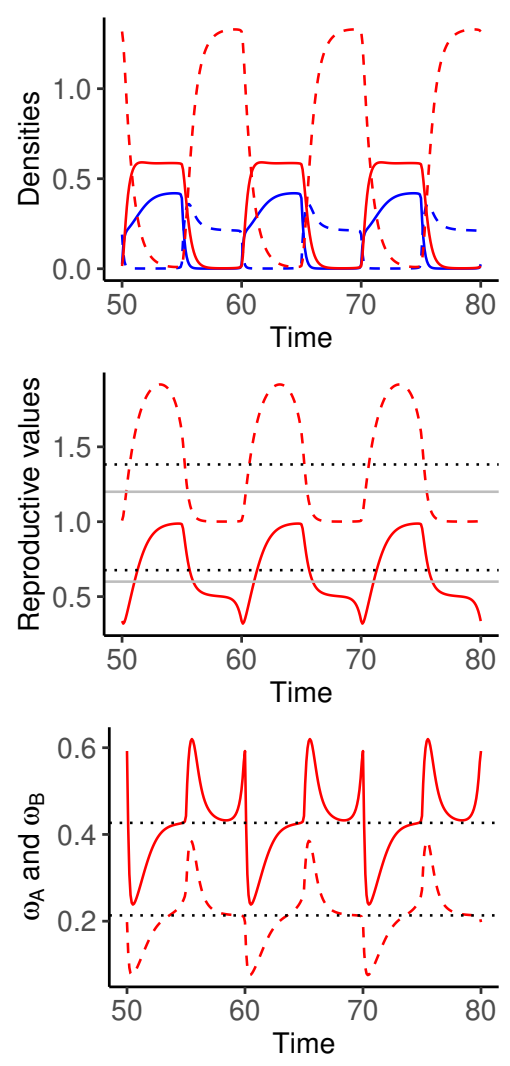

(c) $T=80$
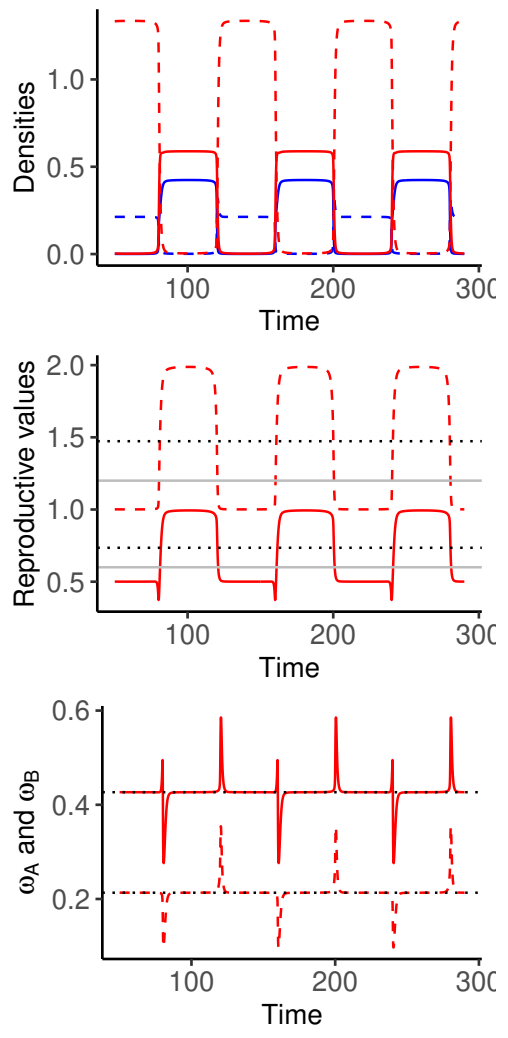

Figure S.4: Scenario 3: imperfect vaccine and virulence. (a) Ecological dynamics on the fast time scale for increasing periods. (a) $T=1$. (b) $T=10$. (c) $T=80$. Top: dynamics of host densities (blue: susceptible hosts, red: infected hosts, solid lines: $A$ hosts, dashed lines: $B$ hosts). Middle: dynamics of individual reproductive values for pathogens in $A$ (solid) and $B$ (dashed) hosts. The gray and dotted lines indicate the equilibrium and mean values respectively. Bottom: dynamics of $\omega^{k}=\left(v^{A} S^{A}+v^{B} S^{B}\right) / v^{k}$ compared to the mean value $\left(d+\alpha^{k}\right) / \beta^{k}$. Parameters: $\nu(t)=0.5(1+(2 / \pi) \arctan (\sin (2 \pi t / T) / 0.01)), b=2, d^{A}=d^{B}=1, \beta^{A}(z)=\beta^{B}(z)=\beta=10 z /(1+z)$, $\alpha^{A}=z, \alpha^{B}=\left(1-r_{a}\right) z, r_{a}=0.8, z_{w}=1.667$. The value of $z_{w}$ is the ESS value in a constant environment $\nu=0.5$. 